ARTICLE

Received 20 Mar 2015 | Accepted 25 Jul 2015 | Published 8 Sep 2015

DOI: $10.1038 /$ ncomms 9163

OPEN

\title{
Involvement of a eukaryotic-like ubiquitin-related modifier in the proteasome pathway of the archaeon Sulfolobus acidocaldarius
}

Rana S. Anjum ${ }^{1, \star}$, Sian M. Bray ${ }^{1, \star}$, John K. Blackwood ${ }^{1}$, Mairi L. Kilkenny ${ }^{1}$, Matthew A. Coelho ${ }^{1, \dagger}$, Benjamin M. Foster ${ }^{1, \dagger}$, Shurong $\mathrm{Li}^{1}$, Julie A. Howard ${ }^{2}$, Luca Pellegrini ${ }^{1}$, Sonja-Verena Albers ${ }^{3, \dagger}$, Michael J. Deery ${ }^{2} \&$ Nicholas P. Robinson ${ }^{1}$

In eukaryotes, the covalent attachment of ubiquitin chains directs substrates to the proteasome for degradation. Recently, ubiquitin-like modifications have also been described in the archaeal domain of life. It has subsequently been hypothesized that ubiquitin-like proteasomal degradation might also operate in these microbes, since all archaeal species utilize homologues of the eukaryotic proteasome. Here we perform a structural and biochemical analysis of a ubiquitin-like modification pathway in the archaeon Sulfolobus acidocaldarius. We reveal that this modifier is homologous to the eukaryotic ubiquitin-related modifier Urm1, considered to be a close evolutionary relative of the progenitor of all ubiquitinlike proteins. Furthermore we demonstrate that urmylated substrates are recognized and processed by the archaeal proteasome, by virtue of a direct interaction with the modifier. Thus, the regulation of protein stability by Urm1 and the proteasome in archaea is likely representative of an ancient pathway from which eukaryotic ubiquitin-mediated proteolysis has evolved.

\footnotetext{
${ }^{1}$ Department of Biochemistry, University of Cambridge, Tennis Court Road, Cambridge CB2 1GA, UK. ${ }^{2}$ Department of Biochemistry and Cambridge Systems Biology Centre, Cambridge Centre for Proteomics, Cambridge CB2 1QR, UK. ${ }^{3}$ Molecular Biology of Archaea, Max Planck Institute for Terrestrial Microbiology, 35043 Marburg, Germany. ${ }^{\star}$ These authors contributed equally to this work. $†$ Present addresses: University of Freiburg, Molecular Biology of Archaea, Institute for Biology II- Microbiology, Schänzlestrasse 1, 79104 Freiburg, Germany (S.-V.A.); The Francis Crick Institute, Euston Road, London, NW1 2BE UK (M.A.C.); Chromatin Biochemistry Group, MRC Clinical Sciences Centre, Imperial College London, Du Cane Road, London W12 ONN, UK (B.M.F.).

Correspondence and requests for materials should be addressed to N.P.R. (email:npr22@cam.ac.uk).
} 
U biquitin (Ub) and the related ubiquitin-like proteins (Ubls), belong to a family of modifiers that covalently attach to a diverse array of cellular targets, and orchestrate a wide variety of regulatory processes in the eukaryotic cell. The role of ubiquitylation (also referred to as ubiquitination) in proteasome-mediated proteolysis pathways has been well characterised $^{1,2}$. In addition to the roles in protein turnover, $\mathrm{Ub}$ and $\mathrm{Ubl}$ conjugation also operates as a post-translational signal to regulate diverse cellular processes and pathways including DNA replication, DNA damage repair, transcription, cell-cycle control, chromatin modification, protein trafficking, autophagy and innate immunity ${ }^{3-7}$.

Ubls were originally thought to be confined to the eukaryotic domain of life. However, the discovery of an unanticipated structural homology between the bacterial sulphur-transfer proteins ThiS and MoaD, and the eukaryotic Ub/Ubl proteins ${ }^{8,9}$ led to speculation that primitive prokaryotic homologues are the antecedents of the eukaryotic $\mathrm{Ub} / \mathrm{Ubl}$ family ${ }^{10}$. These prokaryotic ubiquitin-like homologues share the characteristic Ub/Ubl-like $\beta$-grasp structural fold ${ }^{19-13}$, and are activated with similar catalytic chemistry to $\mathrm{Ub} / \mathrm{Ubls}^{10}{ }^{10}$. However, their primary biological role is to mobilize sulphur during biosynthetic reactions $8,9,14,15$.

The eukaryotic Urm1 (ubiquitin-related modifier-1) protein, which displays both structural and amino-acid sequence homology to the prokaryotic ThiS and MoaD, has been proposed as a candidate for the evolutionary 'missing link' between the eukaryotic $\mathrm{Ub} / \mathrm{Ubl}$ family and the ancestral prokaryotic sulphurtransfer proteins ${ }^{16-23}$. Although ubiquitylation reactions proceed via three well-characterised enzymatic steps, known as the E1-E2-E3 cascade ${ }^{24}$, urmylation events are apparently less complex and only require an E1-like enzyme to generate covalently modified substrates ${ }^{21,22}$. In addition, Urm1 also mediates sulphur mobilization reactions during post-translational tRNA modification reactions ${ }^{25,26}$. This functional duality led to further speculation that some prokaryotic sulphur-transfer Ubl homologues might also play roles in substrate conjugation.

a

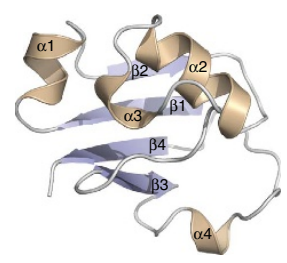

S. solfataricus Urm1

e

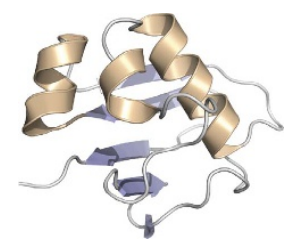

H. volcanii SAMP1

(PDB: 3PO0) b

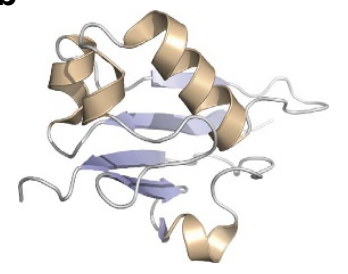

M. musculus Urm1 (PDB: 1XO3)

f

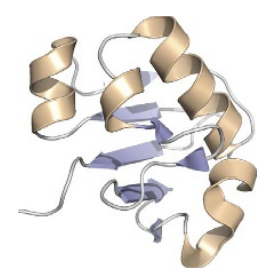

T. thermophilus MoaD (PDB: 1V8C [N-term])
C

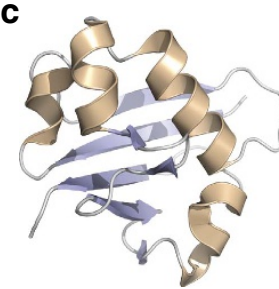

S. cerevisiae Urm1 (PDB: 2QJL)

g

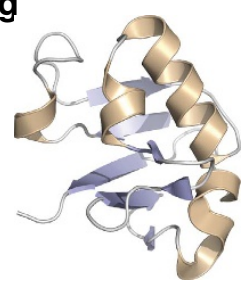

A. orientalis CysO (PDB: 4N6E) d

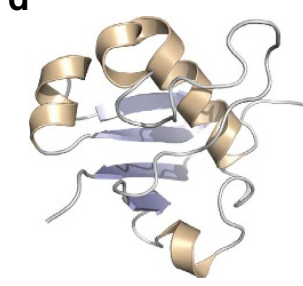

M. acetivorans SAMP1 (PDB: 2L52)

h

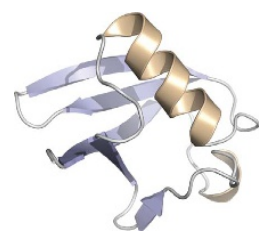

Homo sapiens ubiquitin (PDB: 1UBQ)

Figure 1 | Crystal structure of the S. solfataricus Urm1 protein, and comparison with $\boldsymbol{\beta}$-grasp fold structural homologues. (a) S. solfataricus Urm1 structure shown as a ribbon, and coloured according to secondary structure elements; loops are coloured grey, $\alpha$-helices are shown in wheat, while the $\beta$-sheet is depicted in pale blue (all four $\alpha$-helices and all four $\beta$-strands are numbered). (b-g) Ribbon representation of the M. musculus Urm1, S. cerevisiae Urm1, M. acetivorans SAMP1, H. volcanii SAMP1, T. thermophilus MoaD and A. orientalis CysO $\beta$-grasp fold homologues, respectively; all homologues were identified by DALI and VAST searches. (h) Ribbon representation of $H$. sapiens ubiquitin. All panels are coloured as described in (a). Figures generated using PyMOL ${ }^{68}$. 
the crystal structure of an Urm1/SAMP homologue from the related species $S$. solfataricus, which reveals similarities to both eukaryotic Urm1 proteins, and other archaeal SAMPs. Using tandem mass-spectrometry, we demonstrate that this modifier is conjugated to a variety of targets both in vivo and in vitro. Furthermore, we demonstrated that modified substrates are recognized directly by the $S$. acidocaldarius $20 S$ core proteasome, and by the proteasome-activating nucleotidase (PAN) ATPase ${ }^{44}$. Taken together, our data suggest that Urm1/SAMP modification acts as a signal for substrate recognition by the archaeal proteasome. We discuss our findings in relation to the evolution of Urm1/SAMPs, other Ub/Ubl modifiers, and proteasomal targeting systems.

\section{Results}

Crystal structure of an Urm1 homologue in S. solfataricus. We initially searched for eukaryotic-like Ub and Ubl homologues in the genomes of the thermophilic archaea S. acidocaldarius and S. solfataricus. A eukaryotic Urm1 homologue was identified in both crenarchaeal species by BLAST searches (Supplementary Fig. 1), in agreement with an earlier bioinformatics study ${ }^{33}$. Although Urm1 also shares amino-acid sequence homology with both the Escherichia coli ThiS and MoaD sulphur transfer proteins $^{16}$, PHYRE2 homology searches revealed that these archaeal Urm1 proteins appear more closely related to eukaryotic counterparts, than to the bacterial ThiS/MoaD sulphur transfer components (Supplementary Table 1).

We substantiated our comparison of the archaeal Urm1/SAMP and eukaryotic Urm1 family proteins by determining the crystal structure of S. solfataricus Urm1 at $2.2 \AA$ resolution (Fig. 1a and

\section{Table 1 | Data collection and refinement statistics.}

\begin{tabular}{|c|c|}
\hline & Sso Urm1 \\
\hline \multicolumn{2}{|l|}{ Data collection } \\
\hline Space group & P $22_{1} 2_{1} 2_{1}$ \\
\hline \multicolumn{2}{|l|}{ Cell dimensions } \\
\hline$a, b, c(\AA)$ & 31.4965 .19109 .36 \\
\hline$\alpha, \beta, \gamma\left({ }^{\circ}\right)$ & 909090 \\
\hline Wavelength $(\AA)$ & 1.5418 \\
\hline Resolution $(\AA)$ & $28-2.20(2.30-2.20)^{\star}$ \\
\hline$R_{\text {sym }}$ or $R_{\text {merge }}$ & $0.0642(0.3046)$ \\
\hline$|/ \sigma|$ & $27.26(6.05)$ \\
\hline Completeness (\%) & $99.8(99.3)$ \\
\hline Redundancy & $12.71(8.55)$ \\
\hline Wilson B factor & 25.60 \\
\hline \multicolumn{2}{|l|}{ Refinement } \\
\hline No. reflections & 12.034 \\
\hline $\begin{array}{l}R_{\text {work }} / R_{\text {free }} \\
\text { No. atoms }\end{array}$ & $0.1933 / 0.2278$ \\
\hline Protein & 1,330 \\
\hline Ligand/ion & 5 \\
\hline Water & 89 \\
\hline \multicolumn{2}{|l|}{$B$-factors } \\
\hline Protein & 38.80 \\
\hline Ligand/ion & 92.70 \\
\hline Water & 39.40 \\
\hline \multicolumn{2}{|l|}{ R.m.s. deviations } \\
\hline Bond lengths $(\AA)$ & 0.003 \\
\hline Bond angles $\left({ }^{\circ}\right)$ & 0.66 \\
\hline Ramachandran favoured (\%) & 98.7 \\
\hline Ramachandran outliers (\%) & 0.0 \\
\hline MolProbity Clashscore & 0.36 \\
\hline
\end{tabular}

Supplementary Fig. 2; also see Table 1 for refinement and model statistics). Dali ${ }^{45}$ and VAST ${ }^{46}$ fold recognition searches of the S. solfataricus Urm1 structure revealed homology to a number of eukaryotic Urm1 homologues, as well as to the archaeal $M$. acetivorans and $H$. volcanii SAMP1 proteins (Supplementary Table 2; Fig. 1a-e). Additionally, homology was found to T. thermophilum $\mathrm{MoaD}^{18}$, and the bacterial sulphur transfer protein CysO from Mycobacterium tuberculosis and Amycolatopsis orientalis (Supplementary Table 2). Unlike other bacterial MoaD/ThiS family homologues, the T. thermophilum, and $A$. orientalis proteins displayed exactly the same connectivity of secondary structural elements as the eukaryotic/archaeal Urm1/SAMP modifiers (Fig. 1f,g).

As predicted, the $S$. solfataricus Urm1 adopts a ubiquitin-like $\beta$-grasp fold (Fig. 1h), formed from a four-stranded $\beta$-sheet packed against a central $\alpha$-helix (Fig. 1a). Similar to Ub and other Ubls, the $\beta$-strands are organized in the order $\beta 2-\beta 1-\beta 4-\beta 3$. The inner surface of the sheet, facing the $\alpha$-helix, harbours several buried residues that form the hydrophobic core of the $\beta$-grasp fold. Four $\alpha$-helices are also evident, with the first connecting strands $\beta 1$ and $\beta 2$, the second and third located between strands $\beta 2$ and $\beta 3$, and the final helix positioned on a long loop between strands $\beta 3$ and $\beta 4$ (Fig. 1a). This order of secondary structural features is also conserved in eukaryotic Urml proteins, and in the M. acetivorans SAMP1 structure (PDB:2L52) ${ }^{30}$ (Fig. 1a-d). The bacterial MoaD family of proteins, and the $H$. volcanii SAMP1 fold (PDB:3PO0) ${ }^{31}$, also display the same order of secondary structural elements, up to the final $\alpha$-helix, which is often absent (Fig. 1e $)^{13,47}$. Interestingly, this final helix, linking the last two $\beta$-strands, is a feature shared by the Ub/Ubl and ThiS families ${ }^{13}$.

Inspection of the surface hydrophobicity of the S. solfataricus Urm1 structure revealed the presence of a solvent-exposed hydrophobic patch in close proximity to the C-terminal tail (Fig. 2a). This surface is composed of the conserved hydrophobic residues L52 and L54 on strand $\beta 3$, I77 on strand $\beta 4$, and I79 on the $\mathrm{C}$ terminus of the protein. The conserved residues G8 and I11 also contribute to an adjacent hydrophobic surface, although the K7 lysine residue, conserved across all of the Sulfolobus species, bisects these two solvent-exposed hydrophobic regions (Fig. 2a). Equivalent hydrophobic patches have been described previously in ubiquitin, Urm1 and MoaD, where these surfaces are essential for the interaction with partner proteins, including their cognate E1 activating enzyme $3,18,47$.

A second exposed hydrophobic surface is also present in S. solfataricus Urm1, formed by the residues I19 and V21 on strand $\beta 2$, I33 at the end of helix $\alpha 2$, and F16, on the loop adjoining helix $\alpha 1$ and strand $\beta 2$ (Fig. 2b). Notably the I33 residue, conserved in the Sulfolobales family, was somewhat sunken, creating a shallow hydrophobic depression. This second shallow hydrophobic surface is separate from the first hydrophobic patch, which is located on the opposite face of the central $\beta$-sheet (Fig. 2b). We observed in the structure that a leucine (L67) on the final loop on one Urm1 subunit protrudes into the hydrophobic depression of a neighbouring Urm1 chain to form a dimerization interface (Fig. 2c). However, this arrangement may simply be an artefact as a result of packing in the crystal lattice.

The structure also reveals a narrow hydrophobic channel, juxtaposed to the first hydrophobic patch abutting the C-terminal tail. This trough is lined with the surface exposed conserved residues L27, L47 and G50, and extends from the base of the C-terminal tail to the opposite end of the structure (Fig. 2d). The conserved residues W60 and R61, on helix $\alpha 4$, form one of the sidewalls of this groove, separating it from the nearby hydrophobic patch 1. Interestingly, a similar groove has been reported previously in the M. acetivorans SAMP1 structure (PDB:2L52) ${ }^{30}$, and this feature can also be observed in the eukaryotic Urm1 
a

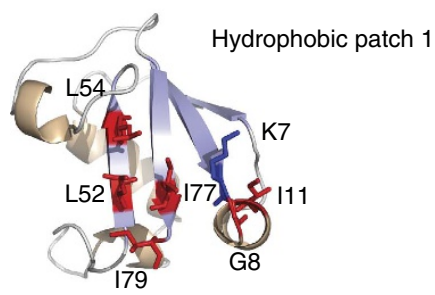

b

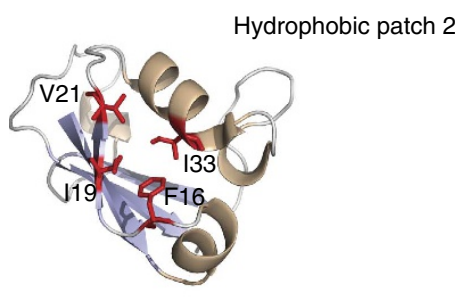

Hydrophobic patch 2
(on both chains)

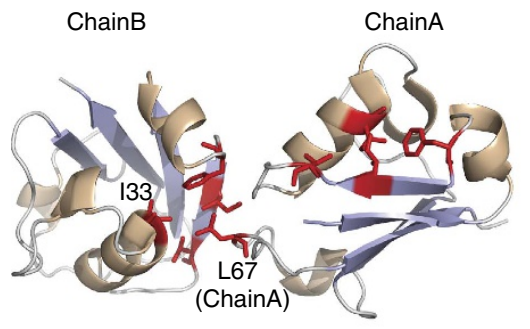

d

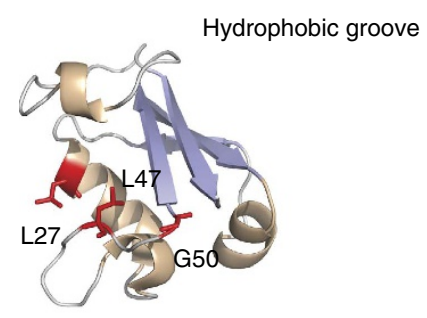

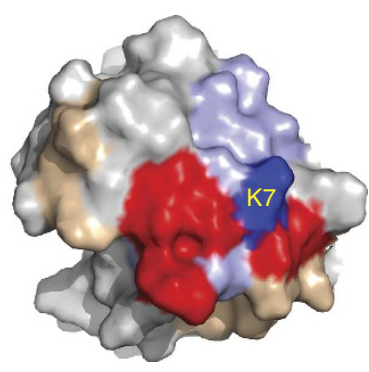
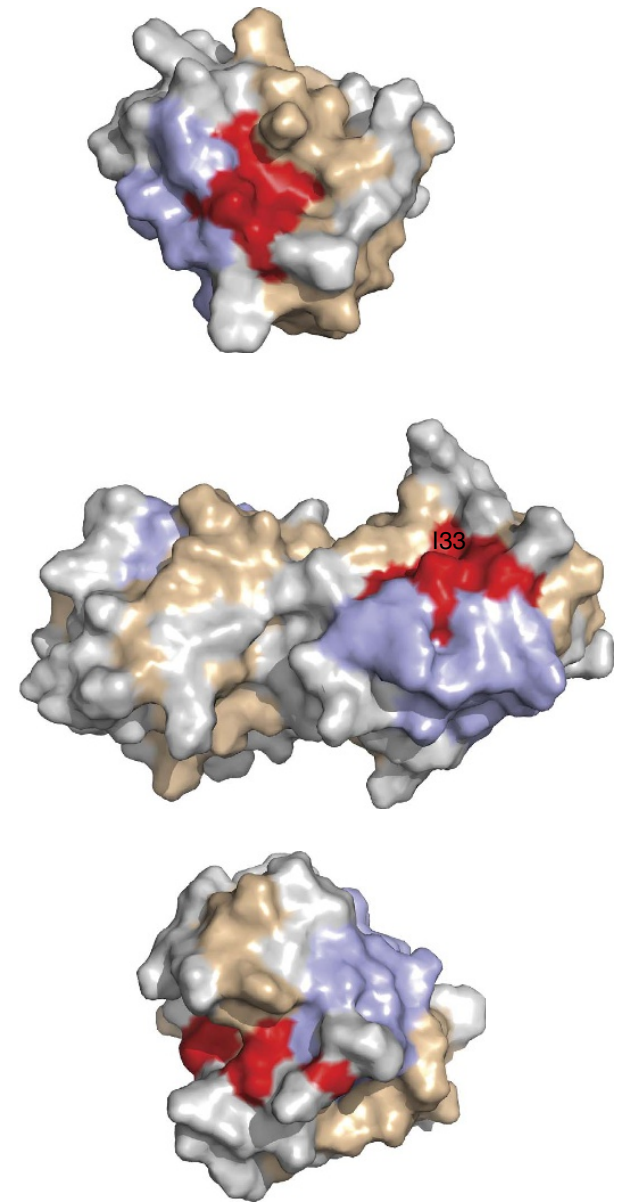

Figure 2 | Architecture of $\boldsymbol{S}$. solfataricus Urm1, identifying exposed hydrophobic surfaces and dimerization interfaces. (a) Left; S. solfataricus Urm1, showing the exposed residues (red sticks) that form the C-terminal exposed hydrophobic patch. The conserved K7 lysine is also shown in dark blue. Right; surface view, identifying the exposed hydrophobic patch 1, coloured in red. (b) Left. view from the opposing edge of the $\beta$-sheet, on Urm 1 chain B, revealing a second hydrophobic patch composed of four exposed residues (F16, I19, V21 and I33; red sticks). Right. Surface view displaying the hydrophobic patch 2, in red. (c) Left: view of Urm1 chains A and B, showing the F16, 119, V21 and I33 patch on both chains. The L67 residue of chain A protrudes into the I33 centred hydrophobic patch on chain B. Right. Surface view of the Urm1 dimer, showing the location of the surface exposed I33 residue on chain A. (d) Left: a hydrophobic groove, lined with the residues G50, L47 and L27 (red sticks) lies adjacent to the C-terminal exposed hydrophobic patch. Right. Surface view of the C-terminal groove. In all panels secondary structure elements are coloured as in Fig. 1 (loops in grey, $\alpha$-helices in wheat, and the $\beta$-sheet in pale blue). Figures generated using $\mathrm{PyMOL}^{68}$.

protein structures (PDB:2AX5) ${ }^{18}$, but is absent from the bacterial MoaD-like family of proteins (PDB:1JW9) ${ }^{47}$. A functional role for this groove has yet to be ascribed in eukaryotic and archaeal Urm1/SAMP homologues.

The Urm1 modifier is activated by an ELSA homologue. All eukaryotic $\mathrm{Ub}$ and $\mathrm{Ubl}$ proteins must be bound and activated by a cognate adenylating enzyme, often referred to as the E1 enzyme. This hydrolyses ATP and catalyses the adenylation of the terminal glycine of the modifier, before substrate conjugation ${ }^{24}$.
In the case of the ancestral MoaD/ThiS sulphur carriers, this step is also necessary for sulphur transfer, and is performed by the MoeB and ThiF E1-like enzymes, respectively ${ }^{14,15}$. Archaeal E1-like/ELSA homologues have been identified previously in both $M$. acetivorans and $H$. volcanii species ${ }^{29,30,48}$. We identified a single ELSA homologue (Saci0179) in the $S$. acidocaldarius genome. This displayed approximately equal sequence identity to both the bacterial ThiF enzyme and also to the eukaryotic Uba4p protein, the E1-like homologue for the eukaryotic urmylation pathway. However, a C-terminal rhodanese domain, harboured in eukaryotic E1 enzymes, was absent in the $S$. acidocaldarius 


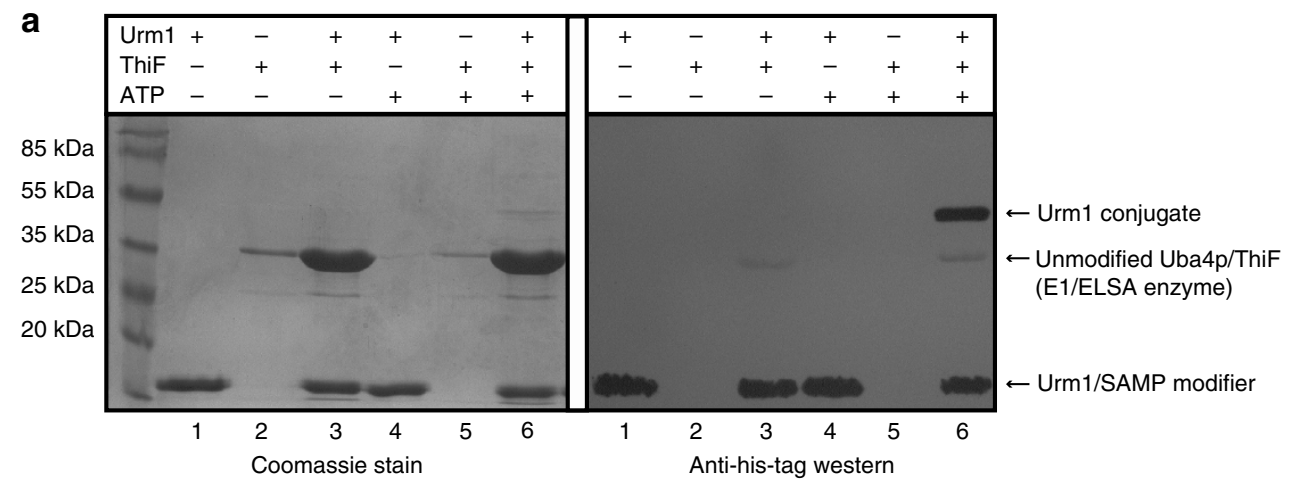

b
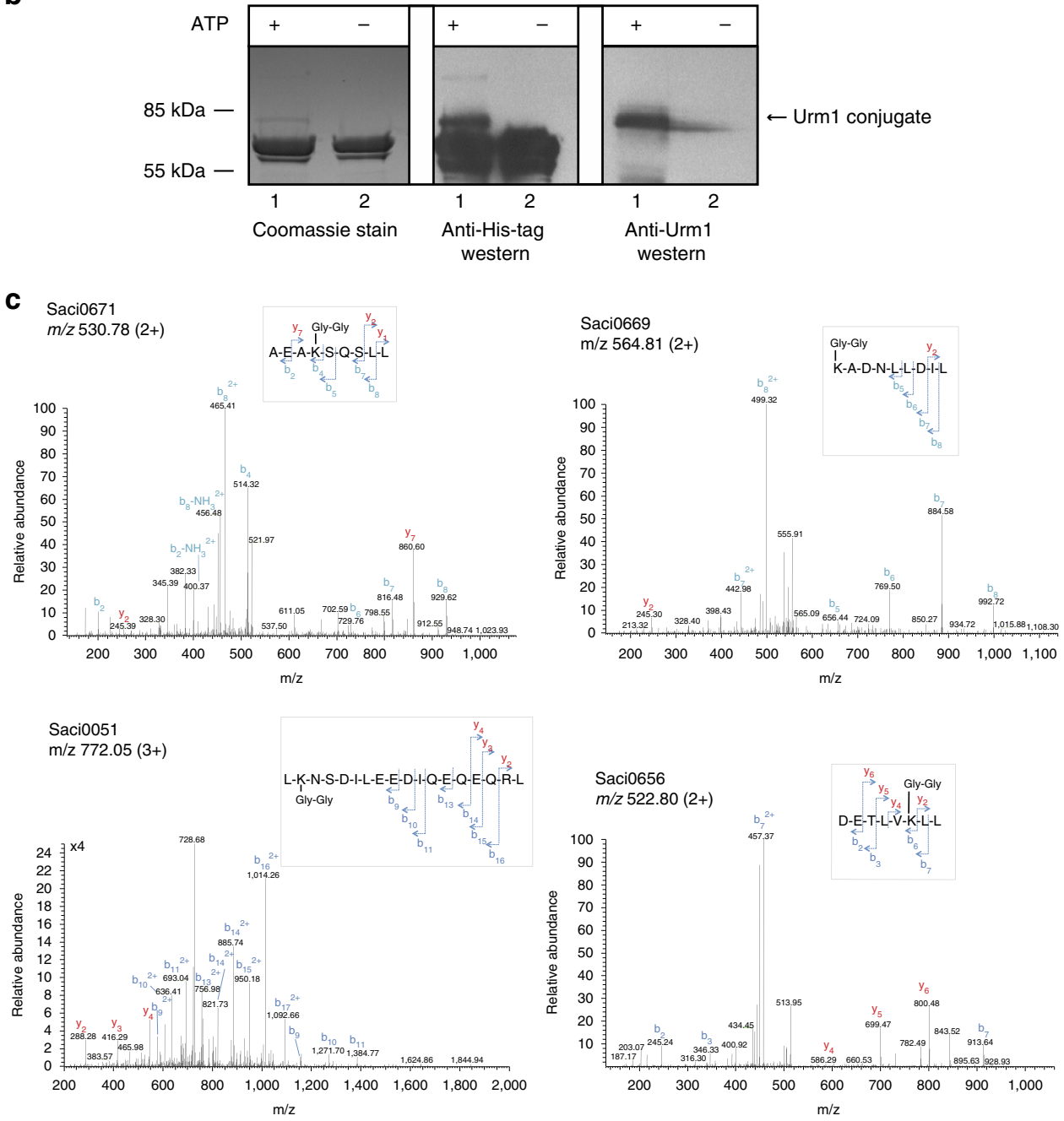

Figure 3 | Production of covalent ATP-dependent Urm1 conjugates by the ELSA/Uba4p/ThiF E1-like enzyme. (a) Auto-urmylation of the ELSA/Uba4p/ThiF enzyme. $30 \mu \mathrm{g} N$-terminally His-tagged Urm1 protein and $90 \mu \mathrm{g}$ ELSA/Uba4p/ThiF enzyme were incubated together at $70{ }^{\circ} \mathrm{C}$ for $1 \mathrm{~h}$ in either the presence or absence of $2.5 \mathrm{mM}$ ATP, and retrieved by Ni-NTA agarose pulldown. Left; Coomassie stained gel of the pull-down. Right; western blot of a duplicate gel, probed with an anti-His antibody. Lanes 1 and 4: Urm1 only controls, without or with ATP, respectively; lanes 2 and 5: untagged ELSA/Uba4p/ThiF only controls, without or with ATP, respectively; lanes 3 and 6: Urm1 plus ELSA/Uba4p/ThiF incubations, without or with ATP, respectively. (b) Urmylation of the Saci0666 thermosome subunit. $30 \mu \mathrm{g}$ N-terminally His-tagged Urm1 protein plus $15 \mu \mathrm{g}$ ELSA/Uba4p/ThiF enzyme and $75 \mu \mathrm{g}$ C-terminally His-tagged Saci0666 were incubated together and pulled-down as described in (a). Lane 1: reaction in the presence of $2.5 \mathrm{mM}$ ATP; lane 2: no ATP control. Left; Coomassie stained gel of the pull-down. Middle and Right; western blot of duplicate gels, probed with an anti-His, or anti-Urm1 antibodies, respectively. (c) Example MS/MS spectra of the diglycine modified peptides from the in vitro urmylation assay (Saci0671 (FBP) and Saci0669 (Urm1)) and in vivo Urm1 overexpression (Saci0051 [Rad50] and Saci0656 [PAN]). $\mathrm{m} / z$ values of the precursor ions are shown in the top left of each panel. $(2+)$ or $(3+)$ indicates doubly or triply charged precursor ions, respectively. Spectra show the annotated peaks that are due to $C$-terminal y (coloured red) and N-terminal b (coloured blue) fragment ions. In each case, the $\mathrm{m} / z$ values of the precursor ions and the $\mathrm{m} / \mathrm{z}$ values of the fragment ions are consistent with diglycine modified lysine residues. The amino-acid sequence of the chymotrypsin-generated peptide, including the di-glycine modified lysine, is shown within the grey box in each example. Other examples are shown in Supplementary Fig. 5. 
enzyme, as has been reported previously for other archaeal and bacterial E1-like proteins ${ }^{29,30,48}$.

To test whether the ELSA/Uba4p/ThiF homologue was indeed the E1 activating enzyme, we purified untagged ELSA/Uba4p/ThiF, and an N-terminally His-tagged Urm1; both $S$. acidocaldarius proteins were expressed heterologously in E. coli. In order to promote complex formation, the two proteins were then incubated together, in either the presence or absence of ATP, before the potential interaction was examined by pull-down assay (Fig. 3a). We observed the untagged ELSA/Uba4p/ThiF associating with the tagged Urm1 protein independently of the addition of ATP. However, the inclusion of the nucleotide resulted in the formation of a conjugate between the two components. Importantly, this conjugate survived boiling in the presence of reducing agent and SDS, indicative of a covalent attachment (Fig. 3a; lane 6). The conjugate observed here is consistent with the previously reported covalent modification of SAMPs in other archaea mediated by the ELSA, following adenylation of the modifier $27,29,30$.

Having demonstrated that the ELSA/Uba4p/ThiF homologue was competent to activate the Urm1 protein, ultimately resulting in the auto-urmylation of the E1-like enzyme, we next examined the ability of the complex to modify an exogenous target protein. Given the strong genomic linkage observed between Urm1 and the $\beta$-subunit of the thermosome (Saci0666) (see Supplementary note and Supplementary Fig. 3), we purified this chaperonin component in E. coli and included the protein in the in vitro Urm1 activation reaction. Addition of this protein in the assay resulted in the formation of a clear ATP-dependent covalent conjugate, confirming that the ELSA/Urm1 complex can modify exogenously added substrates in vitro (Fig. 3b).

Mass spectrometry identification of urmylated substrates. It has been established that Ub, eukaryotic Urm1, and archaeal SAMP conjugates are formed between the C-terminal glycine of the modifier and the $\varepsilon$-amino-group of acceptor lysine residues on the target proteins $s^{1,2,20,24,44}$. A mass spectrometry (MS) approach was therefore adopted to asses whether S. acidocaldarius Urm1 is also covalently attached to substrates by the same linkage. S. acidocaldarius cell-free extract was subjected to an urmylation reaction, using His-tagged Urm1 and untagged ELSA/Uba4p/ThiF enzyme, in the presence of ATP. The resultant His-tagged conjugates were retrieved by pull-down, separated by reducing SDS-polyacrylamide gel electrophoresis (SDS-PAGE), and subsequently digested in-gel with chymotrypsin, before analysis by tandem mass spectrometry (GeLC-MS/MS) (Supplementary Fig. 4A). In parallel to this in vitro approach, we also overexpressed His-tagged Urm1 protein from an exogenous expression vector in S. acidocaldarius cells. The in vivo generated Urm1 conjugates were collected by pull-down following cell disruption, and the resulting material was also analysed by GeLC-MS/MS (Supplementary Fig. 4B). Chymotrypsin treatment resulted in the hydrolysis of the S. acidocaldarius Urm1 peptide backbone between the H82 residue and the penultimate G83 residue of a C-terminal di-glycine motif (G83G84). Hence, if Urm1 was conjugated to a target protein, the chymotrypsin treatment resulted in a fragment harbouring the di-glycine motif, branching from a lysine residue on the linear peptide backbone of the substrate. The addition of a di-glycine motif does not increase the size of the peptides beyond the detection limits of the mass spectrometer, thereby facilitating identification of conjugates.

Analysis of both the in vivo and in vitro urmylation reactions revealed the covalent attachment of the di-glycine motif via an isopeptide bond to the $\varepsilon$-amino-group on a wide variety of substrate lysine residues (Fig. 3c, Supplementary Fig. 5, and Supplementary Table 3A and B). In total, 29 distinct substrate modifications were identified by the in vitro approach, while 25 modifications were observed following the in vivo Urm1 overexpression (Supplementary Table 3A and B). Interestingly, several of these components tallied with the proteins predicted to be involved in urmylation pathways by a bioinformatic analysis of the genomic context surrounding the Urm1 gene (see Supplementary Note and Supplementary Fig. 3). For example, in vitro modifications were identified on the proteasome $\beta$-subunit (Saci0662), and fructose 1,6-bisphosphatase (FBP), while conjugates on a proteasome assembly chaperone (PAC2; Saci0658), PAN (Saci0656), an ATPase RNAaseL inhibitor (RLI), and a HerA/FtsK/TrwB superfamily protein ${ }^{49,50}$ (Saci0667) were observed in the in vivo analysis (Supplementary Table 3A and B). Furthermore, in vivo and in vitro modifications were also detected on several ribosomal subunits, and also on a number of tRNA synthases (Supplementary Table 3A and B). Conjugates were also detected on a number of metabolic enzymes, in addition to FBP, including phosphoglycerate kinase (PGK), phosphoenolpyruvate (PEP) synthase, aldehyde dehydrogenase, alcohol dehydrogenase, and acetyl-CoA synthetase (Supplementary Table 3A and B). Thus, it appears that urmylation may also be involved in the regulation of key metabolic pathways.

The identification of Urm1 modifications on the core $20 \mathrm{~S}$ proteasome, the PAN proteasome regulatory ATPase and the PAC2 proteasome assembly chaperone provided further evidence to suggest a link between urmylation and the proteasome degradation pathway. Modifications were also observed on the Urm1 protein itself in both the in vitro and in vivo analyses (Supplementary Table 3A and B). These findings were suggestive that multiple lysines on Urm1 can be modified, and polymeric

Figure 4 | In vitro reconstitution of the S. acidocaldarius 20 S proteasome. (a) Ni-NTA agarose IMAC chromatography purification step following coexpression of the untagged Saci0613 $\alpha$-subunit with the Saci0662 (His-tagged) and Saci0909 $\beta$-subunits. Lane 1: Saci0613 plus Saci0662; lane 2:

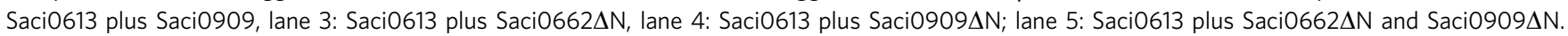
The truncated regions in Saci0662 $\mathrm{N}$ and Saci0909 $\mathrm{N}$, respectively, are indicated by vertical arrows in the schematic below. (b) Size exclusion chromatography (SEC) analysis of the Saci0613/Saci0662 NN/Saci0909 N catalytically active, and Saci0613/Saci0662 $\Delta \mathrm{N}$ inactive 20S proteasome complex. Chromatography UV traces are displayed for both elution profiles. Both peaks are consistent with a complex of $\approx 660 \mathrm{kDa}$. (c) Transmission

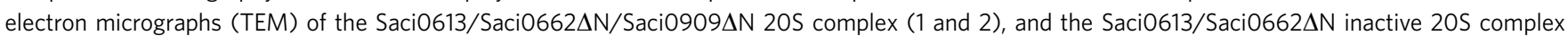
( 3 and 4), negatively stained with uranyl acetate. The left hand panels are at $50000 \mathrm{X}$ magnification and the right hand panels at $\times 100000$ magnification; 100 or $20 \mathrm{~nm}$ scale bars are shown, respectively. (d) Free Urm1 is processed by the active $20 \mathrm{~S}$ proteasome complex, and degradation is stimulated by the addition of the PAN regulatory ATPase. $30 \mu \mathrm{g}$ of Urm1 protein was incubated with $20 \mu \mathrm{g}$ of the proteasome, with or without $20 \mu \mathrm{g}$ of the PAN ATPase complex for $1 \mathrm{~h}$ at $70^{\circ} \mathrm{C}$; all reactions contained $5 \mathrm{mM}$ ATP. Lane 1: Urm1 control with the inactive proteasome; lane 2: Urm1 degradation by the active proteasome; lane 3: Urm1 degradation by the active proteasome stimulated by the PAN ATPase. (e) SEC-MALS analysis of the Saci0613/Saci0662 $\Delta N$ / Saci0909 $\Delta$ N catalytically active $20 \mathrm{~S}$ proteasome. The complex has a fitted molecular weight of $658.1 \mathrm{kDa}( \pm 0.166 \%)$, consistent with a 28 -subunit cylindrical proteasome assembly, with a polydispersity of $1.000( \pm 0.234 \%)$. Differential refractive index $(\mathrm{dRI})$ and light scattering (LS) are plotted in conjunction with molecular weight $\left(M_{w}\right)$. 
chains could be formed, consistent with the SAMP linkages that have been reported previously ${ }^{27-29}$. Since ubiquitin chains have well-established roles in proteasome-mediated degradation, it remains possible that Urm1/SAMP1 chains may also be used for targeting to the proteasome in the archaea. However, it should be noted that the lysine residues modified in this study do not appear to be conserved across archaeal species (Supplementary Table 3A and B; Supplementary Fig. 1).

a

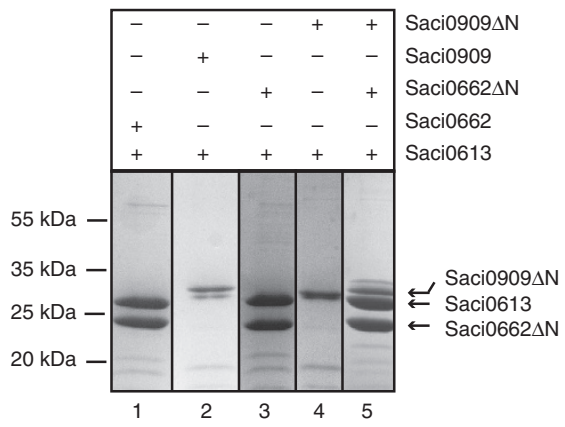

In vitro assembly of the $S$. acidocaldarius $20 S$ proteasome. In order to explore the possible physical and functional association between Urm1 conjugates and archaeal proteasome apparatus we biochemically reconstituted the $20 \mathrm{~S}$ core proteasome from S. acidocaldarius. Archaeal $20 \mathrm{~S}$ proteasomes, like the homologous eukaryotic apparatus, are formed from four stacks of heptameric rings, creating an elongated hollow chamber. Proteasomes from the archaea Thermoplasma acidophilum ${ }^{51,52}$, Archaeoglobus

b

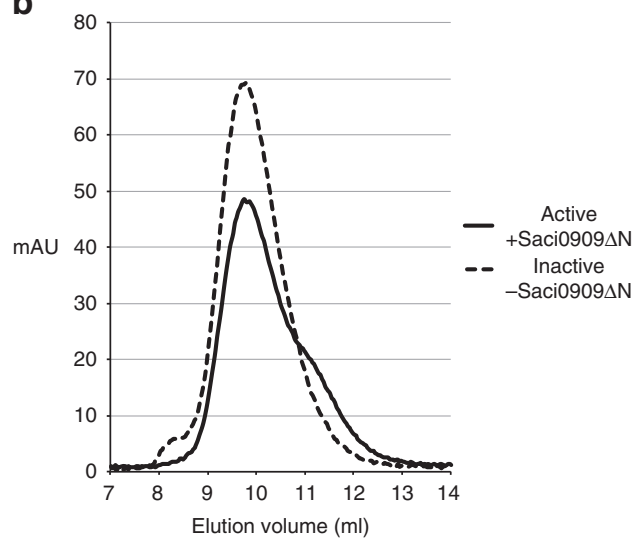

C

Saci0613

+ Saci0909 $\Delta N$ + Saci0662 $\Delta N$ $20 \mathrm{~S}$ proteasomes
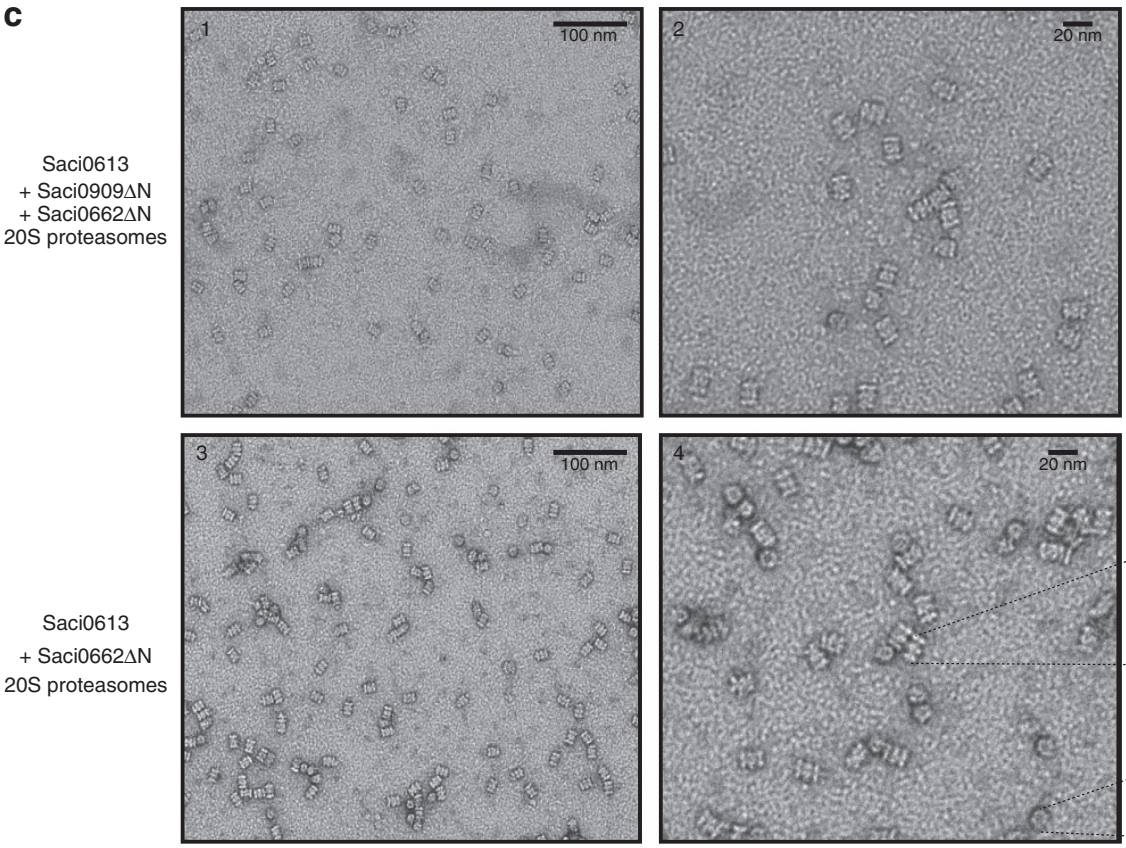

d

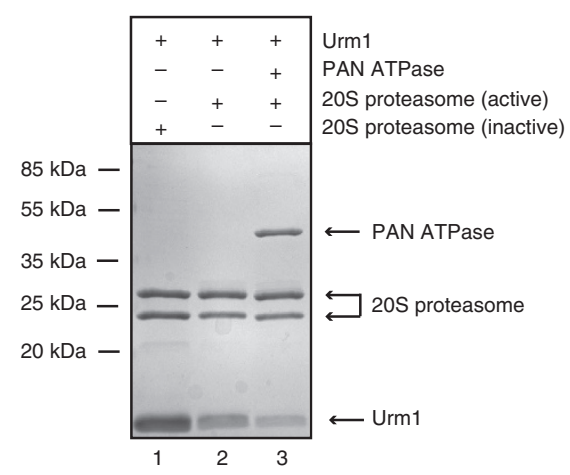

e
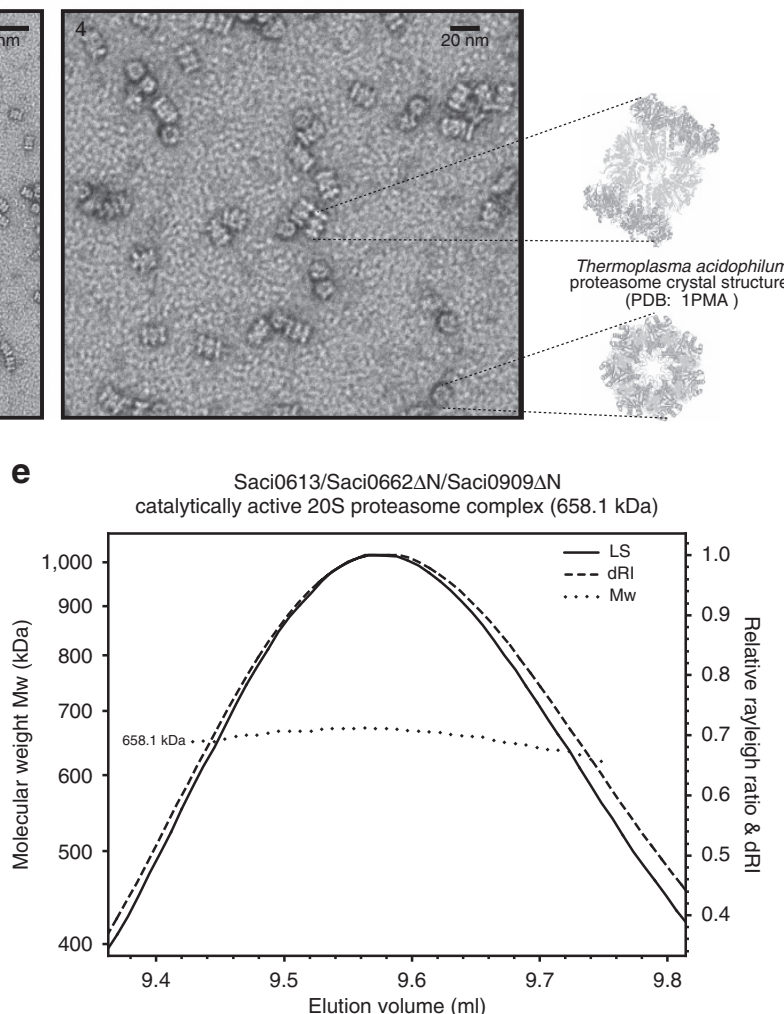
fulgidus $^{53}$ and Methanosarcina thermophila ${ }^{54}$ had previously been reconstituted by recombinant expression in E. coli. In these cases the proteasome is formed from only two $20 \mathrm{~S}$ core proteasome proteins; an $\alpha$-subunit, which produces the selfassembling homo-heptameric $\alpha$-ring outer rings, and a catalytic $\beta$-subunit that constitutes the innermost rings. In contrast, the $S$. acidocaldarius genome encodes two catalytic $\beta$-subunit homologues (Saci0662 and Saci0909), in addition to a single $\alpha$-subunit homologue (Saci0613).

We performed heterologous co-expression trials in $E$. coli with the Saci0613 $\alpha$-subunit, in combination with either the Saci0662 or Saci0909 $\beta$-subunits. In both cases the $\beta$-subunit was C-terminally His-tagged, while the $\alpha$-subunit was native and untagged. We reasoned that if the $20 \mathrm{~S}$ core subunits were expressed with the correct stoichiometry in E. coli then the entire 28-subunit complex should purify over immobilized metal ion affinity chromatography (IMAC). Indeed, a seemingly stoichiometric complex of both $\alpha$ and $\beta$ components was purified following co-expression of Saci0662 with the $\alpha$-subunit. By contrast, the $\alpha$-subunit did not co-elute with the tagged Saci0909 $\beta$-subunit following co-expression and purification over the Ni-NTA agarose column (Fig. 4a). Although the Saci0613 $\alpha / 0662$ $\beta$ proteasome initially appeared stoichiometric, the complex was not stable over size exclusion chromatography (Supplementary Fig. 6). We noted that during $20 \mathrm{~S}$ assembly in both eukaryotic and prokaryotic proteasomes, an $\mathrm{N}$-terminal pro-peptide is commonly cleaved from the $\beta$-subunits to expose a catalytic threonine $(\mathrm{T} 1)^{55}$. Equivalent threonine residues were identified in both of the $S$. acidocaldarius $\beta$-subunits (Fig. 4a), and the $\beta$-subunit expression constructs were therefore redesigned to exclude the preceding $\mathrm{N}$-terminal pro-peptides. The truncated $\beta$-subunits were then co-expressed with the $\alpha$-subunit. As observed with the full-length Saci0909 $\beta$ protein, the truncated version of this $\beta$-subunit failed to form a stable complex with the $\alpha$-subunit (Fig. 4a). In contrast, truncation of the $0662 \beta$-subunit stabilised the 20S assembly, and this material remained stoichiometric during size-exclusion chromatography (Fig. 4b), eluting at a volume consistent with a 28-subunit complex of $660 \mathrm{kDa}$. Furthermore, examination of the purified complex by transmission electron microscopy (TEM) revealed a cylindrical arrangement of four stacked heptameric rings, reminiscent of the overall shape of the previously determined T. acidophilum $20 \mathrm{~S}$ core proteasome crystal structure ${ }^{51}$ (Fig. 4c).

Although co-expression of the $0613 \alpha$-subunit and the truncated $0662 \beta$-subunit resulted in a macromolecular assembly that resembled the structures of the active T. acidophilum, $M$. thermophilus and $H$. volcanii proteasomes ${ }^{51,54,55}$, this material showed no catalytic activity (Fig. 4d). Consequently, both the N-terminally truncated 0662 and $0909 \beta$-subunits were simultaneously co-expressed with the $\alpha$-subunit. This method resulted in formation of another stable complex, again consistent with a 28 -subunit complex, as verified by TEM and SEC-MALS (size-exclusion chromatography-multi-angle laser light scattering) (Fig. 4c,e). However, the complex was now composed of all three subunits, with the Saci0909 $\beta$ subunit incorporating into the $\beta$-subunit ring at a lower ratio than the Saci0662 subunit (Fig. 4a). Critically, the inclusion of the Saci0909 $\beta$-subunit into the $20 \mathrm{~S}$ core conferred catalytic activity to the complex, as demonstrated by the direct degradation of the Urm 1 modifier by the core $20 \mathrm{~S}$ proteasome (Fig. 4d).

Urm1 conjugates engage with the active $20 \mathrm{~S}$ proteasome. Our bioinformatic and mass-spectrometry analyses were suggestive of a link between Urm1 and the proteasome, consistent with previous studies ${ }^{27,33,44}$. However, no categorical demonstration of a physical interaction between an archaeal Ubl and the archaeal $20 \mathrm{~S}$ proteasome has been shown thus far. Having reconstituted the active $S$. acidocaldarius 20 S core proteasome in vitro, we proceeded to perform a series of biochemical assays to explore the potential association between the proteasome and the urmylation pathway. Unexpectedly, we found that the active $20 \mathrm{~S}$ core proteasome complex, with the incorporated catalytic Saci909 $\beta$-subunit, was competent to process the Urm1 protein directly (Fig. 4d). By contrast, we found that a thermally stable (superfolder) green fluorescent protein (GFP) ${ }^{56}$ control protein was not processed under the same conditions (Fig. 5a), suggesting that the Urm1 protein is recognized specifically by the cylindrical protease complex. Furthermore, we also expressed the Saci0656 PAN protein, a homohexameric ATPase homologous to the Rpt hetero-hexameric ring of the eukaryotic proteasome, which interacts with the $\alpha$-ring of the core proteasome ${ }^{57}$. SEC-MALS analysis confirmed the expected hexameric status of this complex (Supplementary Fig. 7). We observed that inclusion of PAN in the assay resulted in a clear stimulation of the Urm1 degradation (Fig. 4d).

After demonstrating that the Urm1 itself is processed by the archaeal proteasome, we next investigated if fusion of Urm1 to a target protein would stimulate degradation of this substrate. $\mathrm{N}$-terminal fusion of Urm1 to the superfolder GFP resulted in substrate association with the $20 \mathrm{~S}$ proteasome and clear degradation of the Urm1 portion of the substrate; crucially the GFP protein was not degraded in the absence of the fused Urm1 tag (Fig. 5a). Furthermore, addition of two or three Urm1 chains, linked in tandem to the GFP substrate, resulted in enhanced substrate processing (Fig. 5a). Circular dicroism (CD) spectroscopy and thermal shift analyses verified that the GFP fusion proteins remained correctly folded, and were not destabilised upon fusion with Urm1 (Fig. 5b,c). In each reaction, an intermediate product was generated, slightly larger than the untagged GFP substrate (Fig. 5a). Mass spectrometry analysis confirmed that this product consisted of a short C-terminal Urm1 peptide attached to the full length GFP protein (Supplementary Fig. 8). This result suggested that the Urm1 tag of these fusion proteins engaged and entered the $20 \mathrm{~S}$ core, but the processing reaction paused, or stalled, as the fused GFP substrate accessed the proteasome (Fig. 5a, Supplementary Fig. 8).

Although it was apparent that the Urm1 region of the GFP fusion proteins were efficiently processed by the active $20 \mathrm{~S}$ core, it was less obvious if a proportion of the fused GFP also entered the $20 \mathrm{~S}$ proteasome. We therefore performed the degradation assays in triplicate using equimolar ratios of the GFP substrates, and then quantified the Coomassie-stained intermediate products, and compared these with the equivalent band in the untagged GFP control. Unexpectedly, this quantification suggested that a proportion of the tagged GFP substrates were also entering and being processed by the active $20 \mathrm{~S}$ core (Fig. 6a,b). This finding was also confirmed by directly measuring the GFP degradation in real-time by following the change in GFP fluorescence throughout the reaction (Fig. 6). It should be noted that in other systems the stably folded GFP proteins are resistant to processing by core proteasomes in the absence of an ATP-driven unfoldase ${ }^{58,59}$. To our knowledge, this is the first time that the S. acidocaldarius $20 \mathrm{~S}$ proteasome has been examined to date, and whereas previous studies using other archaeal 20S cores were performed at $45^{\circ} \mathrm{C}$ (ref. 58), our assays were performed at the more physiological temperature of $70^{\circ} \mathrm{C}$. A control reaction using AMP-PNP (a non-hydrolysable analogue of ATP) ensured that the observed GFP processing was not mediated by a contaminating E. coli ATPase (Supplementary Fig. 9).

We also examined the ability of the PAN regulatory ATPase to recognize and unfold the Urm1-GFP fusion proteins, measuring 

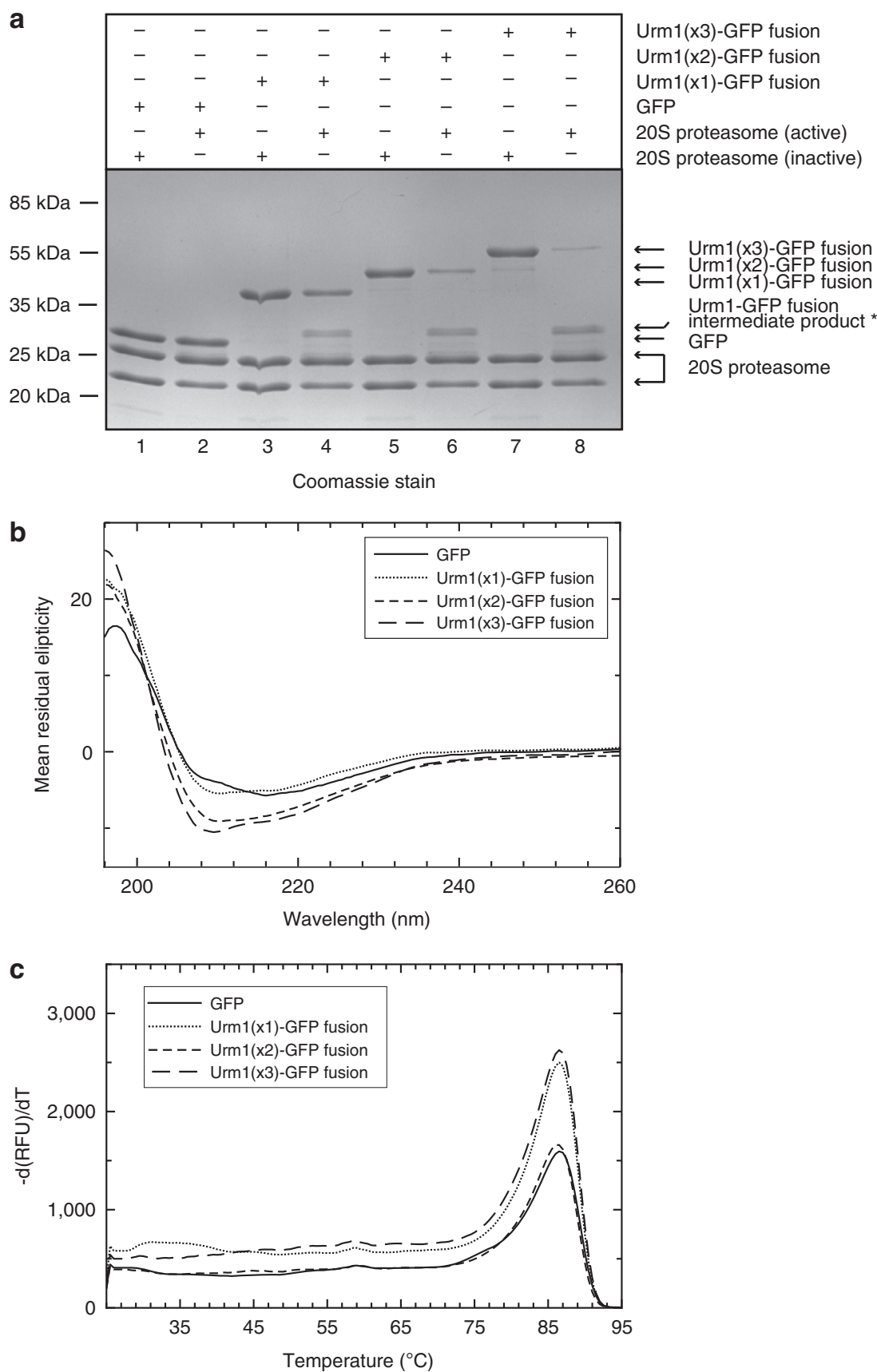

Figure 5 | N-terminal fusion of Urm1 to a GFP substrate results in targeting to the $\mathbf{2 0 S}$ proteasome. (a) N-terminal fusion of Urm1 (or a tandem chain of two, or three, Urm1 subunits) to a thermally stable GFP56 substrate results in processing by the $20 \mathrm{~S}$ proteasome. $10 \mu \mathrm{g}$ of substrate was incubated with $20 \mu \mathrm{g}$ of the proteasome complex for $1 \mathrm{~h}$ at $70^{\circ} \mathrm{C}$. Reactions using the Saci0613/Saci0662 $\Delta \mathrm{N} /$ Saci0909 $\Delta \mathrm{N}$ active complex are shown in lanes 2 (GFP), 4 (1XUrm1:GFP), 6 (2XUrm1:GFP) and 8 (3XUrm1:GFP); lanes 1, 3, 5 and 7: negative controls for each of the GFP substrates, respectively, using the inactive Saci0613/Saci0662 $\Delta \mathrm{N}$ complex. The asterisk denotes an intermediate product in the processing reaction (mass spectrometry analysis of this product is provided in Supplementary Fig. 8). (b) Circular dichroism spectra of GFP and the Urm1:GFP fusion proteins, revealing that Urm1 fusion does not affect GFP folding. The CD spectrum for GFP alone shows negative and positive Cotton effects at about 216 and $198 \mathrm{~nm}$, respectively. This indicates a $\beta$ strand dominated structure as expected. Addition of Urm1 domains to GFP resulted the appearance of another minima at about $208 \mathrm{~nm}$ which is due to the parallel component (relative to $\alpha$-helix axis) of the $\pi$ to $\pi^{\star}$ transition in Urm1 $\alpha$-helices. In addition, there is a shift in the positive cotton effect maximum from $198 \mathrm{~nm}$ towards shorter wavelength, which is probably due to the perpendicular component of the $\pi$ to $\pi^{\star}$ transition in Urm1 $\alpha$-helices. The absence of a clear negative band at $222 \mathrm{~nm}$ due to the $\pi$ to $\pi^{\star}$ transition, which is a signature of $\alpha$-helices, is most likely an indication of the relative small amount of helices in Urm1. (c) Thermal denaturation assay analyses of GFP and the Urm1:GFP fusion proteins, measuring the innate fluorescence of (superfolder) GFP $^{56}$ (490-nm excitation with emission at $510 \mathrm{~nm}$ ). The first derivative plot is displayed (change in relative fluorescence units (RFU) with time [dRFU/ $\mathrm{dT}]$ ). The maximum dRFU/dT occurs at $85^{\circ} \mathrm{C}$ in all samples, indicating that addition of Urm1 to the $\mathrm{N}$ terminus of GFP does not affect the folding or stability of the GFP substrate. 
a

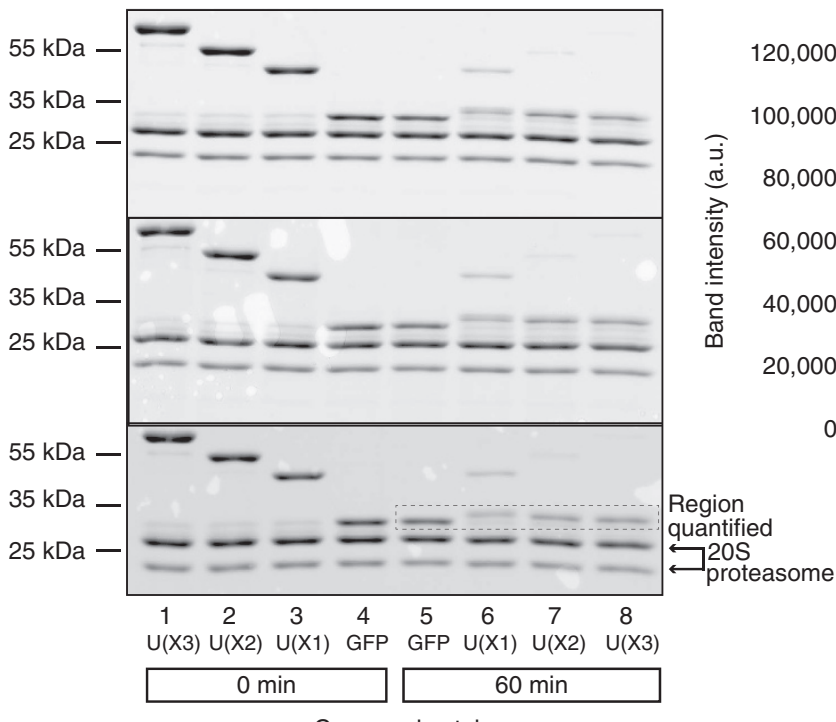

Coomassie stain

C

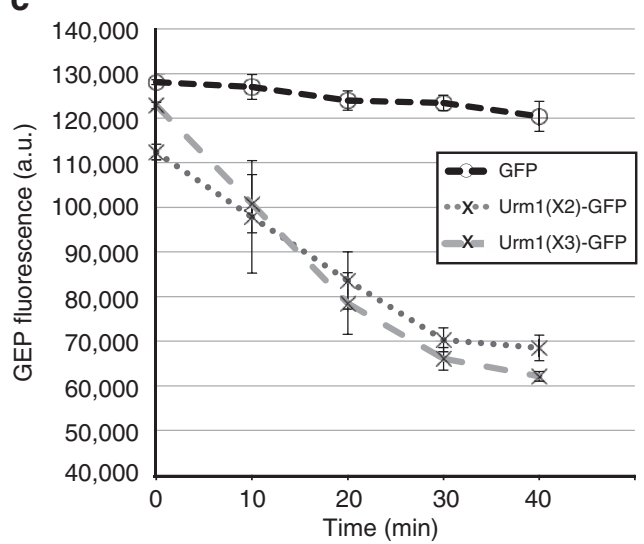

b

d

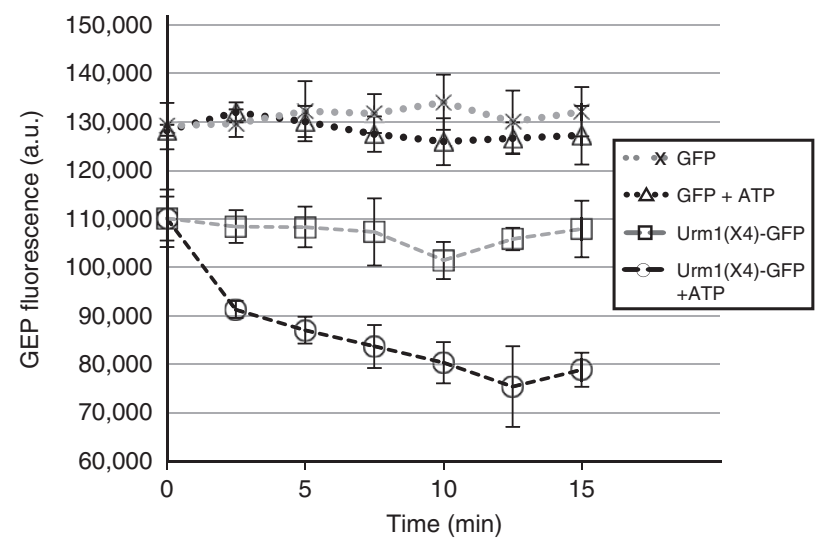

Figure 6 | Quantification of the Urm1-GFP fusion substrate processing by the core 20S proteasome, and unfolding by the PAN ATPase. (a) 205 core processing of the superfolder GFP protein and the 1XUrm1:GFP, 2XUrm1:GFP and 3XUrm1:GFP N-terminal fusion substrates in triplicate and visualized by SDS-PAGE and Coomassie staining. Equimolar quantities $(6 \mu \mathrm{M})$ of the substrates were incubated with $10 \mu g$ Saci0613/Saci0662 $\Delta N /$ Saci0909 $\Delta N$ active $20 \mathrm{~S}$ complex at $69^{\circ} \mathrm{C}$. Lanes $1-4$ display the reactions before heating, while lanes $5-8$ represent the reactions upon termination after 75 min at $69^{\circ} \mathrm{C}$. (b) Quantification of the GFP-sized intermediates (boxed region) after resolution by SDS-PAGE. Gels were scanned using the 'Coomassie Brilliant Blue Digitization' mode on a Typhoon Imaging system (GE Healthcare). The histograms display the mean of three independent repeats and the error bars one s.d. The data were quantified using the ImageQuant software (GE Healthcare) and plotted in Microsoft Excel. (c) Verification of the direct degradation of the Urm1-GFP fusion substrates by the $20 \mathrm{~S}$ core proteasome (at $69^{\circ} \mathrm{C}$ ), demonstrated by the reduction in the GFP fluorescence. (d) ATP-dependent unfolding of a (4X) Urm1:GFP fusion protein by the PAN ATPase (at $60^{\circ} \mathrm{C}$ ) reflected by the decrease in the GFP fluorescence. Untagged GFP is not unfolded by PAN, even in the presence of ATP. In $(\mathbf{c}, \mathbf{d})$ the data points represent the mean of three independent repeats and the error bars one s.d. Data were plotted in Microsoft Excel. The GFP fluorescence was detected at $485 \mathrm{~nm}$ excitation and $520 \mathrm{~nm}$ emission wavelengths, respectively, using a PheraStar (BMG LABTECH) plate reader.

changes in the GFP fluorescence as a readout for substrate unfolding. In contrast to the untagged GFP control, which remained stable even when ATP was included in the reactions, the Urm1-GFP fusion protein was unfolded by the PAN protein in an ATP-dependent manner (Fig. 6d). Taken together, our results provide the first biochemical evidence that Urm1/SAMP tagged substrates are targeted to the archaeal $20 \mathrm{~S}$ proteasome for destruction, by either direct interaction with the $20 \mathrm{~S}$ core or via the PAN regulatory ATPase.

Although ubiquitin may sometimes become conjugated to the $\mathrm{N}$ terminus of substrates in eukaryotes ${ }^{60}$, in addition to the wellcharacterised modifications on internal lysine residues, it is not yet known if Urm1/SAMP proteins can attach to the $\mathrm{N}$ terminus of target proteins in vivo. We therefore performed an in vitro urmylation of the FBP protein, to generate intermediates conjugated to the lysine residues on the substrate and then presented this modified material to the $20 \mathrm{~S}$ proteasomes. While the unmodified FBP protein was not processed by the proteasome (Fig. 7a), the urmylated FBP material associated with the $20 \mathrm{~S}$ core, resulting in the processing of at least the Urm 1 portion of the fused substrate (Fig. 7b); notably this processing was stimulated in the presence of PAN regulatory ATPase (Fig. 7b). Furthermore, an N-terminal fusion of the Urm1-tag to FBP also resulted in targeting of the substrate to the $20 \mathrm{~S}$ core proteasome (Fig. 7c). Again, an intermediate product accumulated indicative of the reaction stalling at the FBP region of the substrate. It therefore appeared that, in the absence of a regulatory unfoldase, the Urm1-FBP fusion substrates stalled at the $20 \mathrm{~S}$ 
a

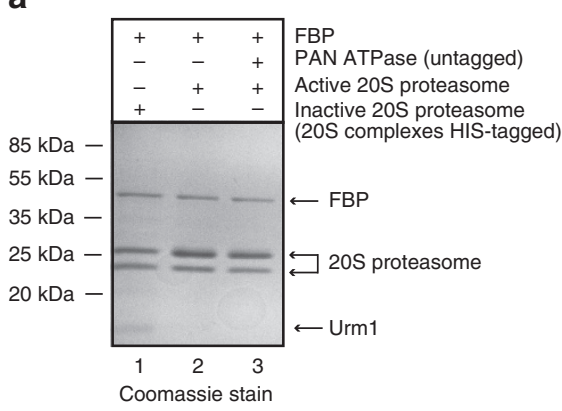

b

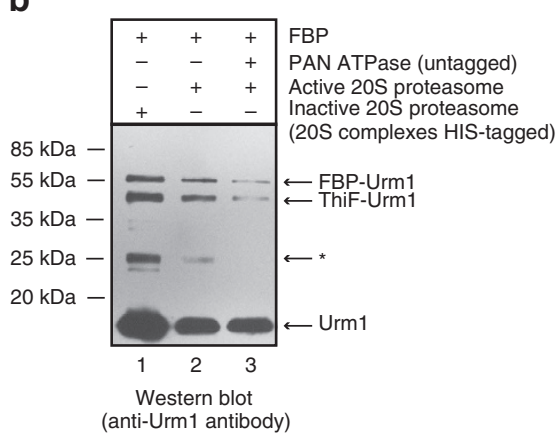

c

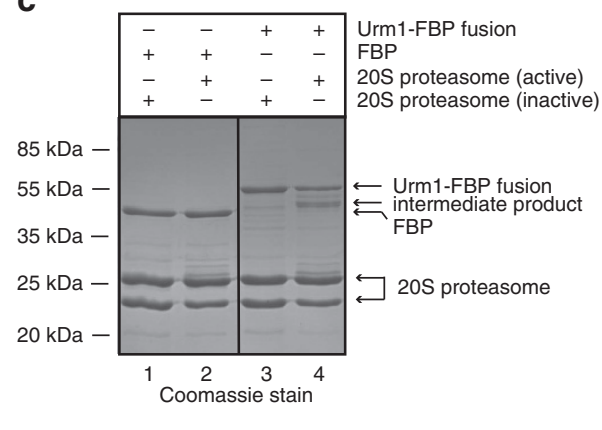

Figure 7 | FPB substrates are directed to the $20 \mathrm{~S}$ proteasome as a result of either Urm1 $\mathbf{N}$-terminal fusion, or by modification on internal lysines using the ELSA/Uba4p/ThiF E1 enzyme. (a,b) In vitro urmylation of FBP and subsequent proteasomal processing by the core 20S and PAN-20S complexes. $100 \mu \mathrm{g} \mathrm{FBP}, 30 \mu \mathrm{g} \mathrm{N}$-terminally His-tagged Urm1 protein and $7.5 \mu \mathrm{g}$ ELSA/Uba4p/ThiF enzyme, plus ATP at $3 \mathrm{mM}$ were incubated together at $70{ }^{\circ} \mathrm{C}$ for $1 \mathrm{~h}$ in a $200 \mu \mathrm{l}$ reaction. $50 \mu \mathrm{l}$ of the urmylated reaction was then incubated at $70^{\circ} \mathrm{C}$ for $1 \mathrm{~h}$ with $20 \mu \mathrm{g} 20 \mathrm{~S}$ proteasome, with or without the addition of $20 \mu \mathrm{g}$ PAN ATPase, and a further $12.5 \mathrm{mM}$ ATP. Products were purified by Ni-NTA pulldown before resolution by SDS-PAGE. (b) Urmylated products were visualized by Western blot probed with an anti-Urm1 antibody. Lane 1: Urmylated FBP reaction incubated with the inactive proteasome (negative control); lane 2: urmylated FBP reaction with processing by the active $20 \mathrm{~S}$ proteasome; lane 3: stimulation of the processing reaction by the active proteasome following addition of the PAN ATPase. Arrows indicate the urmylated FBP product and also an urmylated ELSA/Uba4p/ThiF band. The band visible at $25 \mathrm{kDa}$ (indicated by an asterisk) is an urmylated truncation product of the ELSA/Uba4p/ThiF protein. Panel (a) is a duplicate Coomassie-stained control gel of (b) demonstrating that the non-urmylated FBP substrate is not processed. (c) N-terminal fusion of a single Urm1 moiety to an FBP substrate results in targeting to the $20 \mathrm{~S}$ proteasome. $10 \mu \mathrm{g}$ of substrate was incubated with $20 \mu \mathrm{g}$ of the proteasome complex for $1 \mathrm{~h}$ at $70^{\circ} \mathrm{C}$. Reactions using the active proteasome complex are displayed in lanes 2 (FBP) and 4 (Urm1:FBP). Lanes 1 and 3: negative controls for FBP and Urm1:FBP fusion, respectively, using the inactive proteasome.

proteasome core after efficient degradation of the Urm1 tag, as was observed with the Urm1-GFP proteins.

\section{Discussion}

In eukaryotic cells, the ubiquitin-mediated proteasomal degradation pathway plays critical roles in controlling diverse molecular processes through the regulation of protein turnover ${ }^{1,2}$. Similarly, analogous bacterial systems, using the Pup modifier, target a variety of substrates for destruction at the archaeal/eukaryoticlike 20S proteasomes, found exclusively in the Nitrospirae and Actinobacteria families ${ }^{35-39}$. However, Pup is a functional analogue of ubiquitin ${ }^{43}$, rather than a homologue and seems to have evolved independently from the $\mathrm{Ub} / \mathrm{Ubl}$ family. Since the discovery of the SAMP family of proteins in the archaea ${ }^{27}$, these ancestral Ubls have been implicated in proteasomal processing pathways equivalent to the eukaryotic ubiquitin-proteasome system. However, despite this premise, direct evidence of a physical association between SAMPs and the archaeal proteasome has remained elusive to date.

Our current study has identified a SAMP homologue in $S$. acidocaldarius, which displays remarkable structural and amino-acid sequence similarity to the eukaryotic ubiquitin-like modifier, Urm1. We have revealed that this protein interacts directly with the archaeal $20 \mathrm{~S}$ core proteasome, and is degraded by this protease complex. It should be noted that most natively folded proteins, and even aggregated polypeptides, are generally not processed by $20 \mathrm{~S}$ proteasomes ${ }^{61}$. Therefore, the Urm1 degradation appears to result from a specific recognition of the modifier by the protease complex. Furthermore, the inclusion of the $S$. acidocaldarius PAN regulatory ATPase in this reaction stimulates free Urm1 degradation (Fig. 4d). Crucially we find that covalent attachment of Urm1 to a substrate, either by N-terminal fusion, or by in vitro urmylation on lysine residues of the target, results in recognition of the modified protein directly by the core $20 \mathrm{~S}$ proteasome and also by the PAN regulatory ATPase. Regardless of the attachment site, the Urm1 portion of the conjugate, or fused substrate, was processed directly by the $20 \mathrm{~S}$ core proteasome. However, the larger globular GFP and FBP regions of these substrates accumulated as intermediates during the processing reactions (Figs 5-7). It therefore seems most likely that regulatory unfoldases, such as PAN, are required for the efficient degradation of stably folded globular substrates in $S$. acidocaldarius cells, consistent with previously described energydependent protease complexes in other organisms ${ }^{59}$. Indeed, we observed that the PAN ATPase specifically recognized and unfolded an Urm1-GFP fusion in an ATP-dependent manner (Fig. 6d). It was also clear that the substrates in our assays lacking an Urm1 tag remained refractory to proteasomal processing. Thus, it appears that the covalent conjugation of small modifier proteins to mark substrates for proteasomal destruction is a feature common to eukaryotes and archaea, and also in the $20 \mathrm{~S}$ proteasome-bearing bacterial species.

Unlike the eukaryotic ubiquitin associated degradation system, where the modifier is cleaved from the target and recycled at the proteasome ${ }^{62}$, the archaeal Urm1 protein, is destroyed by the $S$. acidocaldarius core $20 \mathrm{~S}$ proteasome in vitro. Thus, the modifier apparently leads the process, guiding covalently conjugated substrates into the proteasomal cylinder (Fig. 8a). Our quantification of the $20 \mathrm{~S}$ proteasomal processing assays suggested that a proportion of the substrates entered the proteasomal $20 \mathrm{~S}$ core, even in the absence of a regulatory ATPase. This contrasts with previous studies of other archaeal proteasome complexes, and may reflect the fact that our assays are performed at the physiological temperature of $70^{\circ} \mathrm{C}$, rather than the $45^{\circ} \mathrm{C}$ temperature used in earlier investigations $s^{58}$. The mechanism of the Urm1 modifier leading the substrate destruction is highly reminiscent of the bacterial Pupylation pathway, in which the $\mathrm{N}$ terminus of the Pup modifier is first threaded into the core of the protease, via the Mpa regulatory ATPase, leading to the degradation of the modifier and subsequently the covalently attached substrate ${ }^{42}$. Interestingly, deconjugation of Pup from modified targets, by the Dop protein, has been reported previously ${ }^{63,64}$, while SAMPylated proteins in H. volcanii can be de-SAMPylated by JAB1/MPN/MOV34 metalloenzymes ${ }^{65}$. 
a

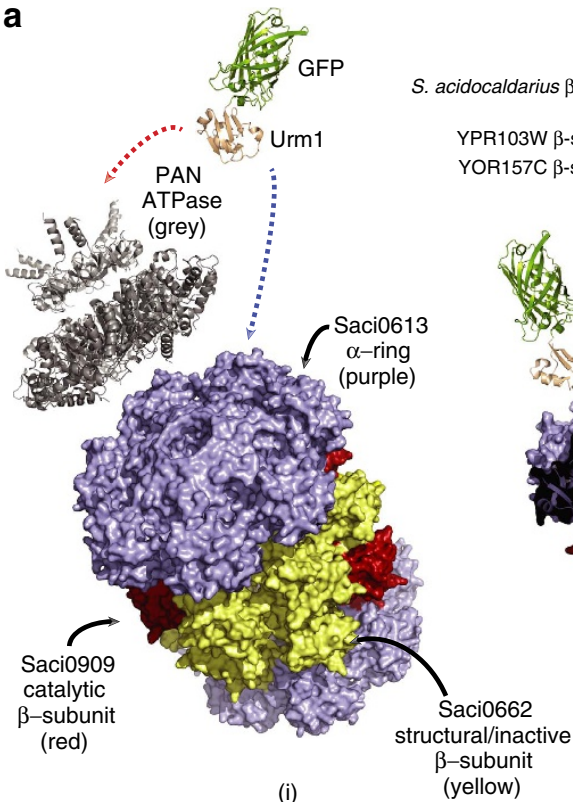

(i) $\beta$-subunit
(yellow)

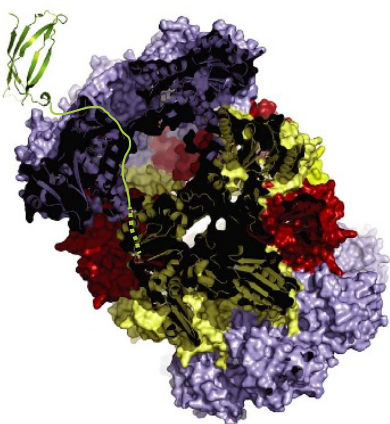

(iii)

b

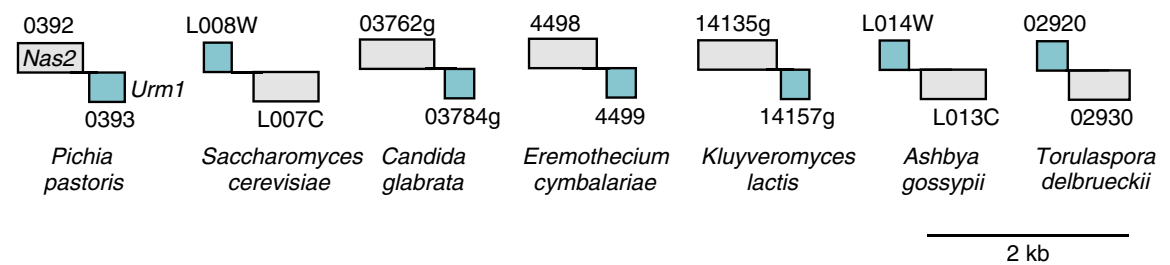

C

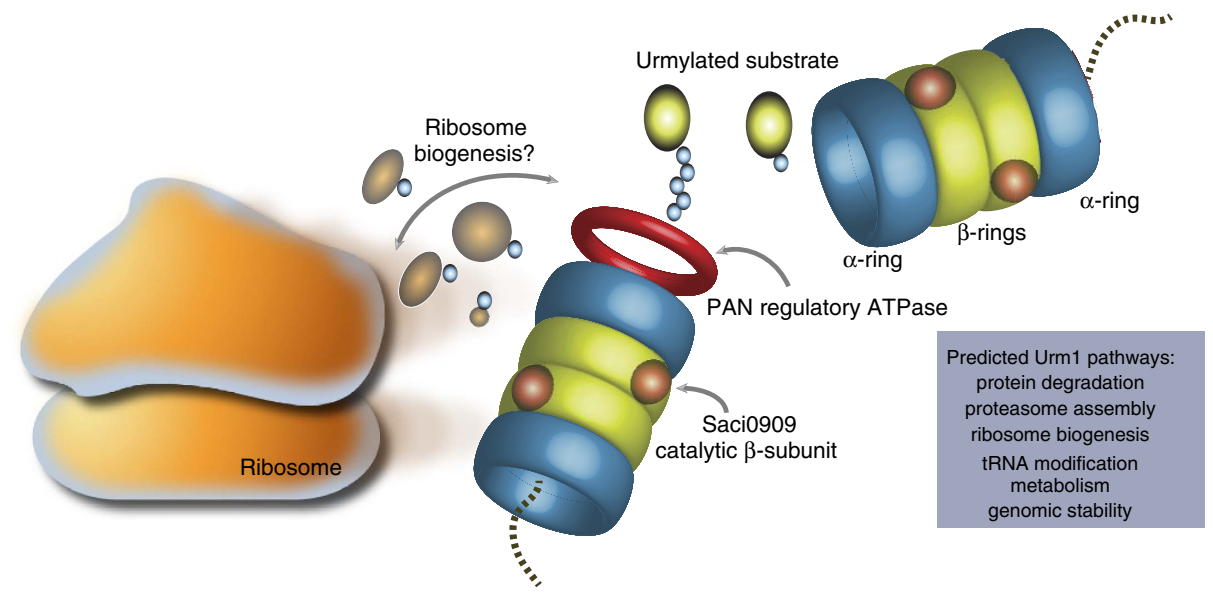

Figure 8 | Proposed models for the archaeal Urm1-proteasome system. (a) Covalent attachment of Urm1 to substrates results in association with either the core 205 proteasome (blue dashed arrow) or the PAN regulatory ATPase (red dashed arrow) in vitro. The Urm1 tag leads the entry of the fused GFP substrate (green) into either complex. (i) For illustrative purposes the T. acidophilum 20 S proteasome crystal structure (PDB:1PMA) is displayed. The 20S outer $\alpha$-rings are coloured purple (equivalent to Saci0613), while the inner $\beta$-rings are shown in yellow (representing Saci0662). Three of the $\alpha$-subunits are coloured red to represent the Saci0909 active catalytic subunit (Inset; yeast homologues of the S.acidocaldarius $\beta$-subunits identified by BLAST, see Supplementary Table 4). The Methanocaldococcus jannaschii PAN complex (grey) is also displayed in a view perpendicular to the central channel (upper domains; the PAN N-terminal hexameric ring (PDB:3H43 (ref. 69)); below; the monomeric PAN nucleotidase domain structure (PDB:3H4M) modelled onto the coordinates of hexameric HsIU (PDB:1DO0) ${ }^{69}$ ); (ii) cutaway view of the proteasome cylinder to demonstrate processing of the Urm1 tag of the Urm1:GFP fusion proteins by the core 20S, in a manner reminiscent of the bacterial Pup-proteasome system; (iii) this study suggests that some stably-folded Urm1tagged substrates may enter the $20 \mathrm{~S}$ core even in the absence of an unfoldase at physiological temperatures. (b) Genomic context associations between eukaryotic Urm1 gene (blue) and the Nas2 proteasome assembly chaperone gene (light grey) in a wide variety of yeast species, suggestive of a putative role for Urm1 in assembly of the eukaryotic proteasome. ORFs positioned above the midline are transcribed left to right, and those below the line transcribed right to left. (c) Schematic model of the archaeal Urm1-proteasome system, and the hypothetical role in the assembly and maintenance of the archaeal ribosome. Urm1 modifications have been detected on several ribosome subunits in this study, while ubiquitin is proposed to play roles in eukaryotic ribosome maintenance $\mathrm{e}^{70}$. Our study also implicates the archaeal Urm1 protein in proteasome assembly pathways, tRNA modification, metabolism, and the maintenance of genomic stability by DNA repair (see Supplementary Note, Supplementary Fig. 3 and Supplementary Tables 3 and 5 for further details). 
Our demonstration of the physical and functional association between the Urm1 protein and the archaeal proteasome necessitated the reconstitution of the $S$. acidocaldarius $20 \mathrm{~S}$ assembly in vitro for the first time. This revealed that the central catalytic $\beta$-rings of this complex are composed of two different subunits. In contrast, the $\beta$-rings of previously purified archaeal 20S proteasomes from $H$. volcanii, T. acidophilum and $M$. thermophilum are homo-heptameric ${ }^{51,54,55}$. In $S$. acidocaldarius, the first of the two $\beta$-subunits is seemingly catalytically inert, and instead appears essential for interaction with the outer $\alpha$-rings to form the 20S cylinder. The second $\beta$-subunit then appears to intersperse within the $\beta$-ring, at a low stoichiometry relative to the other subunits, to provide catalytic activity (Figs 4a,d and 8a). Parallels can been made with the eukaryotic 20S complex in which only three of the seven $\beta$-subunits confer catalytic activity ${ }^{39,52}$. Indeed, BLAST searches reveal that $S$. acidocaldarius catalytic $\beta$-subunit is most similar the $S$. cerevisiae $\beta 5$ catalytic subunit, whereas the other structural $\beta$-subunit is more homologous to the non-catalytic $\beta 7$ in yeasts (Fig. 8a and Supplementary Table 4).

The structural, bioinformatic and functional data presented here add further support to the theory that the archaeal and eukaryotic Urm1 proteins are closely related, and have evolved from a shared common ancestor ${ }^{10}$. However, in eukaryotes, a direct association between the Urm1 modifier and the proteasome complex has not been identified to date. It is clear that the proteasome-mediated degradation pathway has been appropriated by the more sophisticated and adaptable ubiquitylation system in complex eukaryotic organisms ${ }^{24}$. However, has the ancient association of Urm1 with the proteasome that we still observe in the archaeal domain of life been entirely usurped by the ubiquitin system in the eukaryotes? In a final bioinformatic observation, we note that in a variety of yeast species the genes encoding for Urm1 and the Nas2 proteasome assembly chaperone ${ }^{66}$ are immediately juxtaposed (Fig. 8b). This genomic linkage is suggestive of an involvement of Urm1 in the eukaryotic proteasome assembly process. Interestingly, there is some precedent for Urm1 playing a role in the assembly of the archaeal proteasome, as we detected in vivo and in vitro Urm1 modifications on the S. acidocaldarius PAC2 proteasome assembly chaperone (Supplementary Table 3A and $\mathrm{B}$ ). It is tempting to speculate that in the ancestral archaeal system, Urm1 may play roles in both proteasomal assembly and substrate destruction pathways. Similarly, it remains plausible that in eukaryotic organisms Urm1-conjugated proteins could still interact with proteasome, at least during assembly of the protease complex.

The Urm1/SAMP mediated proteasome degradation pathway identified by this study is representative of a streamlined and presumably ancestral version of the eukaryotic ubiquitinproteasome degradation system. It remains to be determined if Urm1 marked substrates are recognized directly by the core $20 \mathrm{~S}$ proteasome in vivo, in accordance with our observations using the in vitro reconstituted S. acidocaldarius system. This may represent an additional processing mechanism operating alongside the established degradation routes that utilize the regulatory ATP-dependent unfoldases, as reported in other systems ${ }^{59}$. Similarly, further studies should also reveal if the conjugation of a single Urm1 protein is sufficient to target substrates to the proteasome in vivo, or whether multiple Urm1 moieties are required for efficient processing. It will also be important to investigate if Urm1 is deconjugated and recycled at the proteasome before substrate delivery, or destroyed in vivo along with the target, as we observe with the reconstituted $20 \mathrm{~S}$ proteasome. Our findings to date are also suggestive that the S. acidocaldarius Urm1 modifier may regulate a variety of diverse cellular processes and pathways (see Supplementary Note and Fig. 8c). Intriguingly, many equivalent biological functions in eukaryotic organisms are also regulated by the Urm1 and Ub modifiers. Thus, the adoption of simple and biochemically tractable archaeal models to investigate ubiquitin-like pathways should provide us with valuable future insights into these key conserved mechanisms.

\section{Methods}

Protein purification. Open reading frames (ORFs) were amplified by PCR from $S$. acidocaldarius DSM639 genomic DNA, cloned into either pET30a, pET33b, pET28a, pCDF-DUET or pET-DUET vectors (Novagen), and expressed in E. coli Rosetta (DE3) pLysS cells (Novagen). Cell lysates were heat-clarified at $70^{\circ} \mathrm{C}$ for $20 \mathrm{~min}$. His-tagged soluble proteins were subsequently purified by Ni-NTA IMAC and size exclusion chromatography. Full experimental details of the cloning strategy and purification procedure for each protein (including the untagged Urm1 and ELSA/Uba4p/ThiF proteins) are provided in the Supplementary Methods and Supplementary Table 6.

Crystallization and X-ray structure determination. The crystal structure of S. solfataricus Urm1 was determined by molecular replacement of a native crystal data set, leading to a complete structure refined to a resolution of $2.2 \AA$. Full details of the structure determination and refinement are given in the Supplementary Methods.

In vitro urmylation assays. Target protein $(75 \mu \mathrm{g}), 30 \mu \mathrm{g} \mathrm{N}$-terminally His-tagged Urm 1 protein and $15-\mu \mathrm{g}$ ELSA/Uba4p/ThiF enzyme were incubated together at $70{ }^{\circ} \mathrm{C}$ for $1 \mathrm{~h}$ in either the presence or absence of 2.5-mM ATP in 200- $\mu \mathrm{l}$ urmylation reaction buffer ( $20 \mathrm{mM}$ Tris acetate ( $\mathrm{pH} 8.0), 100 \mathrm{mM} \mathrm{NaCl}, 5 \mathrm{mM}$ $\mathrm{MnCl}_{2}, 10 \mathrm{mM} \mathrm{MgOAc}, 50 \mathrm{mM} \mathrm{KOAc}, 1 \mathrm{mM} \mathrm{Zn(OAc)}$, $5 \%$ glycerol and $0.02 \%$ $\beta$-mercaptoethanol). Urmylated products were then retrieved by Ni-NTA pulldown, washed in TBST (10 mM Tris ( $\mathrm{pH} 8.0), 150 \mathrm{mM} \mathrm{NaCl}, 0.1 \%$ Tween20) supplemented with $15 \mathrm{mM}$ imidazole, and the products eluted by boiling in 2 laemmli protein loading buffer. Urm 1 conjugates were then separated by SDS-PAGE, and identified by western blot.

\section{In vitro urmylation of S. acidocaldarius cell extract for MS analyses.}

$S$. acidocaldarius cell-free extracts were prepared by collecting 11 of cells at $\mathrm{OD}_{600 \mathrm{~nm}}$ at 0.6 , washed in $1 \mathrm{X}$ TBS $(10 \mathrm{mM}$ Tris $(\mathrm{pH} 8.0), 150 \mathrm{mM} \mathrm{NaCl})$, and resuspended in $5 \mathrm{ml} 1 \mathrm{X}$ TBS, $0.1 \%$ Triton-X-100, $0.1 \% \beta$-mercaptoethanol and $1 \mathrm{X}$ EDTA-free protease inhibitors (Roche). Cells were disrupted by sonication and the lysate clarified by centrifugation. $250 \mu \mathrm{l}$ of the cell-free extract was added to $500-\mu \mathrm{l}$ urmylation reaction buffer, supplemented with $375 \mu \mathrm{g}$ N-terminally His-tagged Urm1 protein, $375 \mu \mathrm{g}$ ELSA/Uba4p/ThiF enzyme and 10-mM ATP, and incubated for $1.5 \mathrm{~h}$ at $70^{\circ} \mathrm{C}$. Urmylated products were purified by Ni-NTA pulldown, separated by SDS-PAGE, and analysed by GeLC-MS/MS

In vivo overexpression of Urm1 for MS analyses. N-terminally hexa-His-tagged Saci0669 (Urm1) was overexpressed in S. acidocaldarius strain MW001 with the vector pCMalLacS ${ }^{67}$, following induction with $0.4 \%$ maltose. Urm 1 conjugates were purified from cell lysate by IMAC on Ni-NTA agarose. Full experimental details are described in the Supplementary Methods.

GeLC-mass spectrometry and MS data analysis. All GeLC-MS/MS experiments were performed using a nanoAcquity UPLC (Waters Corp., Milford, MA) system and an LTQ Orbitrap Velos hybrid ion trap mass spectrometer (Thermo Scientific, Waltham, MA). Full experimental details including sample preparation and data analysis are provided in the Supplementary Methods.

Size exclusion chromatography-multi-angle laser light scattering. Samples analysed by SEC-MALS ( $100 \mu \mathrm{l}$ protein complex at $\left.2 \mathrm{mg} \cdot \mathrm{ml}^{-1}\right)$ were passed over a Superdex 200 10/300 Increase GL column (GE Healthcare), in $20 \mathrm{mM}$ Tris (pH 8.0), $300 \mathrm{mM} \mathrm{NaCl}$. The column output was fed into a DAWN HELEOS II MALS detector with a laser source at $664 \mathrm{~nm}$, and eight fixed angle detectors (Wyatt Technology), followed by an Optilab T-rEX differential refractometer, using a $664 \mathrm{~nm}$ LED light source at $25^{\circ} \mathrm{C}$ (Wyatt Technology).

Reconstitution of the S. acidocaldarius 20 S proteasome. Full length untagged Saci0613 $\alpha$ ORF was coexpressed with N-terminally truncated Saci0662 $\beta$, with or without the $\mathrm{N}$-terminally truncated Saci0909 $\beta$ catalytic subunit in E. coli Rosetta (DE3) pLysS cells (Novagen). A 28 -subunit $(\sim 660 \mathrm{kDa})$ complex was purified by heat clarification of the extract, Ni-NTA agarose IMAC, and size exclusion chromatography. Full experimental details of the cloning strategy and purification procedure are provided in the Supplementary Methods. 
Electron microscopy. S. acidocaldarius 20 S proteasome complexes were visualized with an FEI Philips CM100 transmission electron microscope at the Advanced Imaging Centre, University of Cambridge. $10 \mu \mathrm{l}$ of the $20 \mathrm{~S}$ proteasome complex at $0.035 \mathrm{mg} \mathrm{ml}^{-1}$ was applied to glow-discharged carbon-coated electron microscopy grids, pre-coated with $10 \mu \mathrm{l} 0.1 \%$ poly-L-lysine (Sigma), and negatively stained with $10 \mu \mathrm{l} 2 \%(\mathrm{w} / \mathrm{v})$ uranyl acetate.

$\mathbf{N}$-terminal Urm1 fusion protein proteasome processing assays. Ten microgram of substrate (GFP, Urm1:GFP fusion, FBP, Urm1:FBP fusion or native Urm1 protein) was incubated, for $1 \mathrm{~h}$ at $70^{\circ} \mathrm{C}$, with $20 \mu \mathrm{g}$ of either active (including the Saci0909 catalytic $\beta$ subunit) or inactive (without Saci0909 $\beta$ ) 20S proteasome complex in $70-\mu \mathrm{l}$ proteasome reaction buffer $(20 \mathrm{mM}$ Tris acetate $(\mathrm{pH} 8.0)$, $100 \mathrm{mM} \mathrm{NaCl}, 5 \mathrm{mM} \mathrm{MnCl} 2,10 \mathrm{mM} \mathrm{MgOAc}, 50 \mathrm{mM} \mathrm{KOAc,} 1 \mathrm{mM} \mathrm{Zn}(\mathrm{OAc})_{2}$, $5 \%$ glycerol and $0.02 \% \beta$-mercaptoethanol). After addition of $2 \mathrm{X}$ laemmli protein loading buffer, the products were then separated by SDS-PAGE, and visualized with Coomassie stain. For the quantified assays in Fig. 7 equimolar quantities $(6 \mu \mathrm{M})$ of the GFP, 1XUrm1:GFP, 2XUrm1:GFP and 3XUrm1:GFP were incubated with $10-\mu$ g active $20 \mathrm{~S}$ complex at $69^{\circ} \mathrm{C}$ for $75 \mathrm{~min}$. Gels were scanned using the 'Coomassie Brilliant Blue Digitization' mode on a Typhoon Imaging system (GE Healthcare), and bands were quantified using the ImageQuant software (GE Healthcare).

In vitro urmylation and proteasome processing assay. $100 \mu \mathrm{g} \mathrm{FBP,} 30 \mu \mathrm{g}$ $\mathrm{N}$-terminally His-tagged Urm1 protein and $7.5 \mu \mathrm{g}$ ELSA/Uba4p/ThiF enzyme were incubated together at $70^{\circ} \mathrm{C}$ for $1 \mathrm{~h}$ in 200 - $\mu$ urmylation reaction buffer $(20 \mathrm{mM}$ Tris acetate ( $\mathrm{pH} 8.0$ ), $100 \mathrm{mM} \mathrm{NaCl}, 5 \mathrm{mM} \mathrm{MnCl} 2,10 \mathrm{mM} \mathrm{MgOAc}, 50 \mathrm{mM} \mathrm{KOAc}$, $1 \mathrm{mM} \mathrm{Zn}(\mathrm{OAc})_{2}, 5 \%$ glycerol, $0.02 \% \beta$-mercaptoethanol) and supplemented with $3 \mathrm{mM}$ ATP. $50 \mu \mathrm{l}$ of the urmylated reaction was then incubated, at $70^{\circ} \mathrm{C}$ for $1 \mathrm{~h}$, with $20 \mu \mathrm{g}$ of either catalytically active, or inactive $20 \mathrm{~S}$ proteasome complex, with or without the addition of $20 \mu \mathrm{g}$ of the PAN ATPase, in the presence of an additional $12.5 \mathrm{mM}$ ATP, in a final volume of $80 \mu \mathrm{l}$. Products were then retrieved by Ni-NTA pulldown, washed in TBST ( $10 \mathrm{mM}$ Tris ( $\mathrm{pH} 8.0), 150 \mathrm{mM} \mathrm{NaCl}$ and $0.1 \%$ Tween 20 ) supplemented with $15-\mathrm{mM}$ imidazole. Proteins were eluted by boiling in $2 \mathrm{X}$ laemmli protein loading buffer, separated by SDS-PAGE, and visualized with Coomassie stain.

GFP fluorescence assay measuring 205 degradation and PAN unfolding. For the $20 \mathrm{~S}$ proteasome core degradation reactions, equimolar quantities $(6 \mu \mathrm{M})$ of the GFP, 1XUrm1-GFP, 2XUrm1-GFP and 3XUrm1-GFP were incubated with $10 \mu \mathrm{g}$ active $20 \mathrm{~S}$ proteasome complex in reaction buffer $(20 \mathrm{mM}$ Tris acetate $(\mathrm{pH} 8.0)$, $100 \mathrm{mM} \mathrm{NaCl}, 5 \mathrm{mM} \mathrm{MnCl} 2,10 \mathrm{mM} \mathrm{MgOAc}, 50 \mathrm{mM} \mathrm{KOAc,} 1 \mathrm{mM} \mathrm{Zn(OAc})_{2}$, $5 \%$ glycerol and $0.02 \% \beta$-mercaptoethanol) at $69^{\circ} \mathrm{C}$ for $10,20,30$ or $40 \mathrm{~min}$, respectively. $3 \mu \mathrm{l}$ sample was then diluted in $107-\mu \mathrm{l}$ reaction buffer and $100 \mu \mathrm{l}$ dispensed into the wells of a 96 well 'Half-area' plate (Corning). For the unfolding assays by the PAN ATPase, $4.65 \mu \mathrm{M}$ of a $4 \mathrm{XUrm} 1-\mathrm{GFP}$ fusion protein was incubated with 37- $\mu \mathrm{g}$ PAN in a total reaction buffer, with or without the inclusion of $3.5 \mathrm{mM}$ ATP. Reactions were heated at $60^{\circ} \mathrm{C}$ for $2.5,5,7.5,10,12.5$ and $15 \mathrm{~min}$, respectively, and then spun to remove any aggregates. $3 \mu \mathrm{l}$ sample was then diluted in $107 \mu \mathrm{l}$ reaction buffer and $100 \mu \mathrm{l}$ dispensed into the wells of a 96 well 'Half-area' plate (Corning). For both assays the fluorescence signal was detected using a PheraStar (BMG LABTECH) plate reader, at 485-nm excitation and 520-nm emission wavelengths. The GFP sample, equilibrated at room temperature, was used to calibrate the instrument.

Circular dichroism. CD spectra between 250 and $185 \mathrm{~nm}$ were recorded on an AVIV 410 spectropolarimeter (Aviv Biomedical), at $25^{\circ} \mathrm{C}$ and $0.5 \mathrm{~nm}$ steps. The full methodology is provided in the Supplementary Methods.

Thermal denaturation assay. Melting temperatures were obtained using a 96-well plate format CFX Connect Real Time PCR Detection System (Bio-Rad), equipped with a photodiode detector with FRET channel excitation and emission wavelengths of 490 and $575 \mathrm{~nm}$, respectively, suitable for detection of GFP. Each well contained $5-\mu \mathrm{M}$ protein (as determined by ultraviolet spectroscopy) in $20 \mathrm{mM}$ Tris $\mathrm{Cl}(\mathrm{pH} 8.0), 300 \mathrm{mM} \mathrm{NaCl}, 5 \%$ glycerol and $1 \mathrm{mM}$ DTT buffer in a total volume of $24 \mu \mathrm{L}$. Samples were equilibrated at $25^{\circ} \mathrm{C}$ for $5 \mathrm{~min}$ before increasing the temperature to $95^{\circ} \mathrm{C}$ in $0.5^{\circ} \mathrm{C}$ increments, taking a fluorescence reading after $30 \mathrm{~s}$ settling time with each increment. The fluorescence of the superfolder GFP (Sandia Biotech) fluorophore was followed at $510 \mathrm{~nm}$ as a function of temperature. Melting temperatures was obtained as the lowest point of the first derivative plot (dRFU (relative fluorescence units)/dT), as calculated by the Bio-Rad CFX Manager software (Bio-Rad Laboratories, USA).

\section{References}

1. Hochstrasser, M. Ubiquitin-dependent protein degradation. Annu. Rev. Genet. 30, 405-439 (1996).

2. Hershko, A. \& Ciechanover, A. The ubiquitin system. Annu. Rev. Biochem. 67, 425-479 (1998).
3. Komander, D. \& Rape, M. The ubiquitin code. Annu. Rev. Biochem. 81, 203-229 (2012).

4. Kerscher, O., Felberbaum, R. \& Hochstrasser, M. Modification of proteins by ubiquitin and ubiquitin-like proteins. Annu. Rev. Cell Dev. Biol. 22, 159-180 (2006).

5. Jackson, S. P. \& Durocher, D. Regulation of DNA damage responses by ubiquitin and SUMO. Mol. Cell 49, 795-807 (2013).

6. Teixeira, L. K. \& Reed, S. I. Ubiquitin ligases and cell cycle control. Annu. Rev. Biochem. 82, 387-414 (2013).

7. Van der Veen, A. G. \& Ploegh, H. L. Ubiquitin-like proteins. Annu. Rev. Biochem. 81, 323-357 (2012).

8. Rudolph, M. J., Wuebbens, M. M., Rajagopalan, K. V. \& Schindelin, H. Crystal structure of molybdopterin synthase and its evolutionary relationship to ubiquitin activation. Nat. Struct. Biol. 8, 42-46 (2001).

9. Wang, C., Xi, J., Begley, T. P. \& Nicholson, L. K. Solution structure of ThiS and implications for the evolutionary roots of ubiquitin. Nat. Struct. Biol. 8, 47-51 (2001).

10. Hochstrasser, M. Origin and function of ubiquitin-like proteins. Nature 458, 422-429 (2009).

11. Burroughs, A. M., Balaji, S., Iyer, L. M. \& Aravind, L. Small but versatile: the extraordinary functional and structural diversity of the beta-grasp fold. Biol. Direct 2, 18 (2007).

12. Burroughs, A. M., Iyer, L. M. \& Aravind, L. The natural history of ubiquitin and ubiquitin-related domains. Front. Biosci. (Landmark Ed) 17, 1433-1460 (2012).

13. Iyer, L. M., Burroughs, A. M. \& Aravind, L. The prokaryotic antecedents of the ubiquitin-signaling system and the early evolution of ubiquitin-like beta-grasp domains. Genome Biol. 7, R60 (2006).

14. Taylor, S. V. et al. Thiamin biosynthesis in Escherichia coli. Identification of ThiS thiocarboxylate as the immediate sulfur donor in the thiazole formation. J. Biol. Chem. 273, 16555-16560 (1998).

15. Leimkuhler, S., Wuebbens, M. M. \& Rajagopalan, K. V. Characterization of Escherichia coli MoeB and its involvement in the activation of molybdopterin synthase for the biosynthesis of the molybdenum cofactor. J. Biol. Chem. 276, 34695-34701 (2001).

16. Furukawa, K., Mizushima, N., Noda, T. \& Ohsumi, Y. A protein conjugation system in yeast with homology to biosynthetic enzyme reaction of prokaryotes. J. Biol. Chem. 275, 7462-7465 (2000).

17. Goehring, A. S., Rivers, D. M. \& Sprague, Jr G. F. Urmylation: a ubiquitin-like pathway that functions during invasive growth and budding in yeast. Mol. Biol. Cell 14, 4329-4341 (2003).

18. Xu, J. et al. Solution structure of Urm1 and its implications for the origin of protein modifiers. Proc. Natl Acad. Sci. USA 103, 11625-11630 (2006).

19. Schmitz, J. et al. The sulfurtransferase activity of Uba4 presents a link between ubiquitin-like protein conjugation and activation of sulfur carrier proteins. Biochemistry 47, 6479-6489 (2008)

20. Pedrioli, P. G., Leidel, S. \& Hofmann, K. Urm1 at the crossroad of modifications. 'Protein Modifications: Beyond the Usual Suspects' Review Series. EMBO Rep. 9, 1196-1202 (2008).

21. Van der Veen, A. G. et al. Role of the ubiquitin-like protein Urml as a noncanonical lysine-directed protein modifier. Proc. Natl Acad. Sci. USA 108, 1763-1770 (2011).

22. Petroski, M. D., Salvesen, G. S. \& Wolf, D. A. Urm1 couples sulfur transfer to ubiquitin-like protein function in oxidative stress. Proc. Natl Acad. Sci. USA 108, 1749-1750 (2011).

23. Wang, F., Liu, M., Qiu, R. \& Ji, C. The dual role of ubiquitin-like protein Urm1 as a protein modifier and sulfur carrier. Protein Cell 2, 612-619 (2011).

24. Hochstrasser, M. Biochemistry. All in the ubiquitin family. Science 289, 563-564 (2000).

25. Chowdhury, M. M., Dosche, C., Lohmannsroben, H. G. \& Leimkuhler, S. Dual role of the molybdenum cofactor biosynthesis protein MOCS3 in tRNA thiolation and molybdenum cofactor biosynthesis in humans. J. Biol. Chem. 287, 17297-17307 (2012).

26. Schlieker, C. D., Van der Veen, A. G., Damon, J. R., Spooner, E. \& Ploegh, H. L. A functional proteomics approach links the ubiquitin-related modifier Urm1 to a tRNA modification pathway. Proc. Natl Acad. Sci. USA 105, 18255-18260 (2008).

27. Humbard, M. A. et al. Ubiquitin-like small archaeal modifier proteins (SAMPs) in Haloferax volcanii. Nature 463, 54-60 (2010).

28. Miranda, H. V. et al. Archaeal ubiquitin-like SAMP3 is isopeptide-linked to proteins via a UbaA-dependent mechanism. Mol. Cell Proteomics 13, 220-239 (2014).

29. Miranda, H. V. et al. E1- and ubiquitin-like proteins provide a direct link between protein conjugation and sulfur transfer in archaea. Proc. Natl Acad. Sci. USA 108, 4417-4422 (2011).

30. Ranjan, N., Damberger, F. F., Sutter, M., Allain, F. H. \& Weber-Ban, E. Solution structure and activation mechanism of ubiquitin-like small archaeal modifier proteins. J. Mol. Biol. 405, 1040-1055 (2011). 
31. Jeong, Y. J., Jeong, B. C. \& Song, H. K. Crystal structure of ubiquitin-like small archaeal modifier protein 1 (SAMP1) from Haloferax volcanii. Biochem. Biophys. Res. Commun. 405, 112-117 (2011).

32. Li, Y. et al. Crystal structure of the ubiquitin-like small archaeal modifier protein 2 from Haloferax volcanii. Protein Sci. 22, 1206-1217 (2013).

33. Makarova, K. S. \& Koonin, E. V. Archaeal ubiquitin-like proteins: functional versatility and putative ancestral involvement in tRNA modification revealed by comparative genomic analysis. Archaea 2010, pii: 710303, doi:10.1155/2010/ 710303 (2010).

34. Barandun, J., Delley, C. L. \& Weber-Ban, E. The pupylation pathway and its role in mycobacteria. BMC Biol. 10, 95 (2012)

35. Pearce, M. J., Mintseris, J., Ferreyra, J., Gygi, S. P. \& Darwin, K. H. Ubiquitin-like protein involved in the proteasome pathway of Mycobacterium tuberculosis. Science 322, 1104-1107 (2008).

36. Mayr, J., Seemuller, E., Muller, S. A., Engel, A. \& Baumeister, W. Late events in the assembly of 20S proteasomes. J. Struct. Biol. 124, 179-188 (1998).

37. Zuhl, F., Seemuller, E., Golbik, R. \& Baumeister, W. Dissecting the assembly pathway of the 20S proteasome. FEBS Lett. 418, 189-194 (1997)

38. Raju, R. M., Goldberg, A. L. \& Rubin, E. J. Bacterial proteolytic complexes as therapeutic targets. Nat. Rev. Drug Discov. 11, 777-789 (2012).

39. Forster, F., Unverdorben, P., Sledz, P. \& Baumeister, W. Unveiling the long-held secrets of the 26S proteasome. Structure 21, 1551-1562 (2013)

40. Katayama, Y. et al. The two-component, ATP-dependent Clp protease of Escherichia coli. Purification, cloning, and mutational analysis of the ATP-binding component. J. Biol. Chem. 263, 15226-15236 (1988).

41. Kessel, M. et al. Homology in structural organization between E. coli ClpAP protease and the eukaryotic 26S proteasome. J. Mol. Biol. 250, 587-594 (1995).

42. Striebel, F., Hunkeler, M., Summer, H. \& Weber-Ban, E. The mycobacterial Mpa-proteasome unfolds and degrades pupylated substrates by engaging Pup's $\mathrm{N}$-terminus. EMBO J. 29, 1262-1271 (2010).

43. Barandun, J., Delley, C. L., Ban, N. \& Weber-Ban, E. Crystal structure of the complex between prokaryotic ubiquitin-like protein and its ligase PafA. J. Am. Chem. Soc. 135, 6794-6797 (2013).

44. Maupin-Furlow, J. Proteasomes and protein conjugation across domains of life. Nat. Rev. Microbiol. 10, 100-111 (2012).

45. Holm, L. \& Sander, C. Protein structure comparison by alignment of distance matrices. J. Mol. Biol. 233, 123-138 (1993).

46. VAST (Vector Alignment Search Tool). Available at http:// www.ncbi.nlm.nih.gov/Structure/VAST/vastsearch.html.

47. Lake, M. W., Wuebbens, M. M., Rajagopalan, K. V. \& Schindelin, H. Mechanism of ubiquitin activation revealed by the structure of a bacterial MoeB-MoaD complex. Nature 414, 325-329 (2001).

48. Burroughs, A. M., Iyer, L. M. \& Aravind, L. Natural history of the E1-like superfamily: implication for adenylation, sulfur transfer, and ubiquitin conjugation. Proteins 75, 895-910 (2009).

49. Rzechorzek, N. J. et al. Structure of the hexameric HerA ATPase reveals a mechanism of translocation-coupled DNA-end processing in archaea. Nat Commun. 5, 5506 (2014).

50. Iyer, L. M., Makarova, K. S., Koonin, E. V. \& Aravind, L. Comparative genomics of the FtsK-HerA superfamily of pumping ATPases: implications for the origins of chromosome segregation, cell division and viral capsid packaging. Nucleic Acids Res. 32, 5260-5279 (2004).

51. Lowe, J. et al. Crystal structure of the $20 \mathrm{~S}$ proteasome from the archaeon $\mathrm{T}$. acidophilum at 3.4A resolution. Science 268, 533-539 (1995).

52. Seemuller, E. et al. Proteasome from Thermoplasma acidophilum: a threonine protease. Science 268, 579-582 (1995).

53. Groll, M., Brandstetter, H., Bartunik, H., Bourenkow, G. \& Huber, R. Investigations on the maturation and regulation of archaebacterial proteasomes. J. Mol. Biol. 327, 75-83 (2003).

54. Maupin-Furlow, J. A., Aldrich, H. C. \& Ferry, J. G. Biochemical characterization of the $20 \mathrm{~S}$ proteasome from the methanoarchaeon Methanosarcina thermophila. J. Bacteriol. 180, 1480-1487 (1998).

55. Maupin-Furlow, J. A., Wilson, H. L., Kaczowka, S. J. \& Ou, M. S. Proteasomes in the archaea: from structure to function. Front Biosci. 5, D837-D865 (2000).

56. Pedelacq, J. D., Cabantous, S., Tran, T., Terwilliger, T. C. \& Waldo, G. S. Engineering and characterization of a superfolder green fluorescent protein. Nat. Biotechnol. 24, 79-88 (2006).

57. Bar-Nun, S. \& Glickman, M. H. Proteasomal AAA-ATPases: structure and function. Biochim. Biophys. Acta. 1823, 67-82 (2012).

58. Benaroudj, N., Zwickl, P., Seemuller, E., Baumeister, W. \& Goldberg, A. L. ATP hydrolysis by the proteasome regulatory complex PAN serves multiple functions in protein degradation. Mol. Cell 11, 69-78 (2003).

59. Sauer, R. T. \& Baker, T. A. AAA + proteases: ATP-fueled machines of protein destruction. Annu. Rev. Biochem. 80, 587-612 (2011).

60. Ciechanover, A. \& Ben-Saadon, R. N-terminal ubiquitination: more protein substrates join. in. Trends Cell Biol. 14, 103-106 (2004).

61. Wenzel, T. \& Baumeister, W. Conformational constraints in protein degradation by the 20 S proteasome. Nat. Struct. Biol. 2, 199-204 (1995).
62. Reyes-Turcu, F. E., Ventii, K. H. \& Wilkinson, K. D. Regulation and cellular roles of ubiquitin-specific deubiquitinating enzymes. Annu. Rev. Biochem. 78, 363-397 (2009).

63. Burns, K. E. et al. 'Depupylation' of prokaryotic ubiquitin-like protein from mycobacterial proteasome substrates. Mol. Cell 39, 821-827 (2010).

64. Imkamp, F. et al. Dop functions as a depupylase in the prokaryotic ubiquitin-like modification pathway. EMBO Rep. 11, 791-797 (2010).

65. Hepowit, N. L. et al. Archaeal JAB1/MPN/MOV34 metalloenzyme (HvJAMM1) cleaves ubiquitin-like small archaeal modifier proteins (SAMPs) from protein-conjugates. Mol. Microbiol. 86, 971-987 (2012).

66. Satoh, T. et al. Structural basis for proteasome formation controlled by an assembly chaperone nas2. Structure 22, 731-743 (2014).

67. Berkner, S., Wlodkowski, A., Albers, S. V. \& Lipps, G. Inducible and constitutive promoters for genetic systems in Sulfolobus acidocaldarius. Extremophiles 14, 249-259 (2010).

68. The PyMOL Molecular Graphics System, Version 1.5.0.4 Schrödinger, LLC https://www.pymol.org/.

69. Zhang, F. et al. Structural insights into the regulatory particle of the proteasome from Methanocaldococcus jannaschii. Mol. Cell 34, 473-484 (2009).

70. Shcherbik, N. \& Pestov, D. G. Ubiquitin and ubiquitin-like proteins in the nucleolus: multitasking tools for a ribosome factory. Genes Cancer 1, 681-689 (2010).

\section{Acknowledgements}

N.P.R. would like to thank Helen Wise for initial discussions regarding ubiquitin-like modifications in the archaea. S.M.B., R.S.A., J.K.B. and N.P.R. are grateful to Jeremy Skepper and colleagues at the Cambridge Advanced Imaging Centre for assistance and advice with the TEM. N.P.R. and R.S.A would like to thank Joseph Maman and Katherine Stott for their assistance with the CD and thermal shift analyses of the Urm1:GFP fusion proteins, and Tommaso Moschetti for advice with the plate-reader assays. We would also like to thank Len Packman (Protein \& Nucleic Acid Chemistry (PNAC) Facility, Department of Biochemistry, Cambridge University) for the mass spectrometry analysis of the Urm1:GFP proteasomal processing assay. N.P.R would also like to thank Steve Jackson for his critical reading of the manuscript, and Neil Rzechorzek for assistance and advice with the preparation of the PyMOL illustrations, and superimposition of the hexameric PAN model. N.P.R. is grateful to the Department of Biochemistry and the Isaac Newton Trust (Trinity College, Cambridge) for supporting this study. The N.P.R. laboratory is funded by the Medical Research Council [Career Development Award G0701443], and also by an Isaac Newton Trust Research Grant. S.M.B. is supported by a BBSRC Doctoral Training Grant [RG53842]. L.P. and M.L.K were funded by a Wellcome Trust Senior Fellowship Award in Basic Biomedical Sciences [grant no. 08279/Z/07/Z]. S.V.A. was supported by intramural funds of the Max Planck Society. X-ray diffraction data were collected at the Crystallographic X-ray facility at the Department of Biochemistry, University of Cambridge; we are grateful to the Facility Manager, Dr Dimitri Chirgadze, for his assistance in using these facilities.

\section{Author contributions}

N.P.R. designed the study, collected and analysed the data and wrote the manuscript. S.M.B., R.S.A., J.K.B., M.L.K., M.A.C., B.M.F., S.L., J.A.H., M.J.D. and N.P.R. collected and analysed the data. M.A.C. and M.L.K. crystallised and solved the structure of S. solfataricus Urm1. J.A.H. and M.J.D. collected the mass-spectrometry datasets. S.V.A. provided overexpression vectors and gave assistance with the Urm1 in vivo overexpression.

\section{Additional information}

Accession codes: The coordinates and structure factors of the S. solfataricus Urm1 crystal structure have been deposited in the Protein Data Bank under accession code 4WWM

Supplementary Information accompanies this paper at http://www.nature.com/ naturecommunications

Competing financial interests: The authors declare no competing financial interests

Reprints and permission information is available online at http://npg.nature.com/ reprintsandpermissions/

How to cite this article: Anjum, R. S. et al. Involvement of a eukaryotic-like ubiquitin-related modifier in the proteasome pathway of the archaeon Sulfolobus acidocaldarius. Nat. Commun. 6:8163 doi: 10.1038/ncomms9163 (2015).

This work is licensed under a Creative Commons Attribution 4.0 International License. The images or other third party material in this article are included in the article's Creative Commons license, unless indicated otherwise in the credit line; if the material is not included under the Creative Commons license, users will need to obtain permission from the license holder to reproduce the material. To view a copy of this license, visit http://creativecommons.org/licenses/by/4.0/ 